Article

\title{
Longitudinal Dynamics of Human B-Cell Response at the Single-Cell Level in Response to Tdap Vaccination
}

\author{
Indu Khatri ${ }^{1,2}{ }^{\oplus}$, Annieck M. Diks ${ }^{1}$, Erik B. van den Akker $\left.{ }^{2,3}{ }^{(}\right)$, Liesbeth E. M. Oosten ${ }^{4}$, Jaap Jan Zwaginga ${ }^{4} \mathbb{D}$, \\ Marcel J. T. Reinders ${ }^{2,5} \mathbb{D}$, Jacques J. M. van Dongen ${ }^{1, * \mathbb{D}}$ and Magdalena A. Berkowska ${ }^{1}$ \\ 1 Department of Immunology, Leiden University Medical Center, 2333 ZA Leiden, The Netherlands; \\ i.khatri@lumc.nl (I.K.); A.M.Diks@lumc.nl (A.M.D.); M.A.Berkowska@lumc.nl (M.A.B.) \\ 2 Leiden Computational Biology Center, Leiden University Medical Center, 2333 ZC Leiden, The Netherlands; \\ e.b.van_den_akker@lumc.nl (E.B.v.d.A.); M.J.T.Reinders@tudelft.nl (M.J.T.R.) \\ 3 Department of Molecular Epidemiology, Leiden University Medical Center, 2333 ZC Leiden, The Netherlands \\ 4 Department of Hematology, Leiden University Medical Center, 2333 ZA Leiden, The Netherlands; \\ L.E.M.Oosten@lumc.nl (L.E.M.O.); j.j.zwaginga@lumc.nl (J.J.Z.) \\ 5 Delft Bioinformatics Lab, Delft University of Technology, 2628 CD Delft, The Netherlands \\ * Correspondence: J.J.M.van_Dongen@lumc.nl
}

\section{check for}

updates

Citation: Khatri, I.; Diks, A.M.; van den Akker, E.B.; Oosten, L.E.M.; Zwaginga, J.J.; Reinders, M.J.T.; van Dongen, J.J.M.; Berkowska, M.A. Longitudinal Dynamics of Human B-Cell Response at the Single-Cell Level in Response to Tdap Vaccination. Vaccines 2021, 9, 1352. https://doi.org/10.3390/ vaccines 9111352

Academic Editor: François Meurens

Received: 8 October 2021

Accepted: 13 November 2021

Published: 18 November 2021

Publisher's Note: MDPI stays neutral with regard to jurisdictional claims in published maps and institutional affiliations.

Copyright: (c) 2021 by the authors. Licensee MDPI, Basel, Switzerland. This article is an open access article distributed under the terms and conditions of the Creative Commons Attribution (CC BY) license (https:/ / creativecommons.org/licenses/by/ $4.0 /)$.

\begin{abstract}
To mount an adequate immune response against pathogens, stepwise mutation and selection processes are crucial functions of the adaptive immune system. To better characterize a successful vaccination response, we performed longitudinal (days 0, 5, 7, 10, and 14 after Boostrix vaccination) analysis of the single-cell transcriptome as well as the B-cell receptor (BCR) repertoire (scBCR-rep) in plasma cells of an immunized donor and compared it with baseline B-cell characteristics as well as flow cytometry findings. Based on the flow cytometry knowledge and literature findings, we discriminated individual B-cell subsets in the transcriptomics data and traced over-time maturation of plasmablasts/plasma cells (PB/PCs) and identified the pathways associated with the plasma cell maturation. We observed that the repertoire in PB/PCs differed from the baseline B-cell repertoire e.g., regarding expansion of unique clones in post-vaccination visits, high usage of IGHG1 in expanded clones, increased class-switching events post-vaccination represented by clonotypes spanning multiple IGHC classes and positive selection of CDR3 sequences over time. Importantly, the Variable gene family-based clustering of BCRs represented a similar measure as the gene-based clustering, but certainly improved the clustering of BCRs, as BCRs from duplicated Variable gene families could be clustered together. Finally, we developed a query tool to dissect the immune response to the components of the Boostrix vaccine. Using this tool, we could identify the BCRs related to anti-tetanus and anti-pertussis toxoid BCRs. Collectively, we developed a bioinformatic workflow which allows description of the key features of an ongoing (longitudinal) immune response, such as activation of PB/PCs, Ig class switching, somatic hypermutation, and clonal expansion, all of which are hallmarks of antigen exposure, followed by mutation \& selection processes.
\end{abstract}

Keywords: B-cell receptor repertoire; Tdap vaccine; pertussis; single cell; vaccination

\section{Introduction}

The adaptation of the immune system to recognize antigenically distinct epitopes of pathogens is a critical feature of the antibody $(\mathrm{Ab})$-mediated immunity in response to infection/vaccination. Abs are products of terminally differentiated B cells, plasma cells (PCs), and are the secreted forms of B-cell receptors (BCRs). Generation of a highly diverse $\mathrm{BCR}$ repertoire (BCR-rep) starts during precursor B-cell development in bone marrow, where developing B cells assemble immunoglobulin heavy (IGH) and light chain genes in the process of $V(D) J$ recombination. B cells with a functional BCR leave the bone marrow and are ready to recognize antigens in the peripheral immune system. Following antigen recognition, the BCR undergoes affinity maturation in the germinal centers (GCs) through 
introduction of somatic hypermutations (SHM) and subsequent selection processes for B cells with high-affinity BCR [1]. The introduction and subsequent selection of SHM are critical steps in raising the appropriate immune response. Typically, through positive selection, SHM accumulate in so called complementarity-determining regions (CDRs) because CDRs are in direct contact with the antigen, while SHM in the framework regions (FWR) are less frequently selected, because FWRs are responsible for maintaining receptor structure. Additionally, B cells optimize their effector functions by exchanging the initial $\mathrm{C} \mu$ constant region by other downstream IGH constant regions. This process is known as class-switch recombination (CSR). Memory B cells that have recognized pathogen/antigen circulate through the peripheral lymphoid system, differentiate into plasmablasts and re-enter bone marrow to become long-lived Ab-producing PCs.

The immune response to a booster vaccination or a re-encounter of the same antigen would lead to the use of pre-existing Abs as the first line of defense [2]. The encounter with an antigen will initiate the processes of SHM and affinity maturation where new Abs are generated from the pool of the pre-existing memory B cells $[3,4]$. Using flow cytometry, we have recently shown that in booster vaccination settings (Boostrix), an increase in circulating PC numbers, associated with their phenotypical maturation, occurs as early as fivedays post-vaccination, with a peak in expansion and maturation at day seven post-vaccination [5]. However, the dynamics of the over-time changes in the BCRrep after Boostrix vaccination in humans are not known yet. Therefore, we performed longitudinal measurements and analysis of single-cell transcriptomics and single-cell BCRrep (scBCR-rep) in PCs derived from an individual vaccinated with a Tdap Booster vaccine (Boostrix ${ }^{\circledR}$, GlaxoSmithKline), and compared them with the total BCR-rep at baseline. Along with understanding the dynamics of scBCR-rep after Boostrix vaccination, with this pilot study, we aimed to assess the differences between the flow cytometry and single-cell transcriptomics data at the level of protein vs. transcript expression level and develop a bioinformatics workflow to identify key features of an ongoing (longitudinal) immune response. Furthermore, we also tracked the footprints of the selection processes e.g., $V(D) J$ usage, level of SHMs in FWRs and CDRs and CSR events in B-cell clonotypes to understand the immune responses in a booster vaccination.

As the Boostrix vaccine contains multiple antigens i.e., diphtheria toxoid (DT), tetanus toxoid (TT), three Bordetella pertussis proteins i.e., filamentous hemagglutinin (FHA), pertactin (Prn) and pertussis toxoid (PT), and aluminum hydroxide as an adjuvant [6], we obtained receptor repertoire information against all components of the vaccine. Although it is known that the majority of PCs in the response peak at day 7 (d7) are Ag-specific [7,8], we aimed to dissect the immune response against each component. This was, however, subjected to the known pool of Abs against the antigens of Boostrix vaccine. Knowing limitations of the available anti-toxoid Abs for toxoids in vaccine, we developed a method to search the known $\mathrm{Ab} C \mathrm{CDR} 3$ sequences for these vaccine components in our data.

Here, we have analyzed the longitudinal single-cell transcriptomics and scBCR-rep data in PCs derived from an individual vaccinated with Boostrix vaccine and compared them with the total B cells and/or plasma cells at baseline. Using transcriptomics data, we have identified additional membrane markers for flow cytometry that can be used to identify the B-cell subtypes and maturation of plasma cells. With the scBCR-rep data, we have clustered functionally similar receptors and assessed different components of the repertoire for associations between visits and to baseline. Importantly, we found BCRs associated with the vaccine components in scBCR-rep data.

\section{Methods}

\subsection{Inclusion Criteria and Informed Consent from the Subject}

The donor used in this study was a 28-year-old female, who was recruited as one of 10 healthy adults in a vaccination study where volunteers received Boostrix and wherein their immune responses were monitored over-time by means of flow cytometry (registration number: P16-214, EUDRACT: 2016-002011-18). This longitudinal study aimed at under- 
standing the (Ag-specific) cellular immune responses post-Boostrix vaccination and was approved by the Medical Ethics Committee Leiden-Den Haag-Delft. Inclusion and exclusion criteria were previously published [5]. In short, the donor had to be generally healthy, had completed a vaccination schedule according to the Dutch National Immunization program (www.rivm.nl/en/national-immunisation-programme; accessed on 31 August 2020) and had no known or suspected exposure to pertussis infection nor vaccination in the past 10 years. She also provided an informed consent prior to the inclusion. The individual was selected for the current single-cell study as she had a representative increase in plasma cells at the peak of the plasma cell response (i.e., day 7 (d7). EDTA-blood samples for single-cell transcript sequencing were collected at baseline and d5, d7, d10 and d14 post-vaccination, which based on the parent study, corresponded to the plasma cell expansion (d5-d10) and contraction of plasma cell response [5] (Figure S1). The sequencing of the samples of only one individual were performed as a pilot study to optimize the pipeline to sort cells from the frozen samples, to make comparative assessment of the flow cytometry and transcriptomics data and to develop a pipeline for downstream processing the BCR-rep data and identify toxoid specific BCRs from total B cells.

\subsection{High Throughput B-Cell and Plasmablast/Plasma Cell Sorting}

Prior to the sort, 78.2 million PBMCs from five different time-points were individually (per time-point) stained with an Ab cocktail for surface markers for 30 min at RT in the dark (CD19 BV786, CD24 BV650, CD27 BV421, CD38 PerCP-Cy5.5), followed by a live/dead staining (Zombie NIR Fixable Viability kit, Biolegend) according to the manufacturer's protocol. Next, samples were prepared for multiplexing according to the protocols provided on the 10x Genomics website (www.10xgenomics.com; accessed on 9 January 2019). In short, Fc block was added and after a $10 \mathrm{~min}$ incubation at $4{ }^{\circ} \mathrm{C}$, unique cell hashing $\mathrm{Abs}$ (10x Genomics) were added to each sample separately (per time-point). After incubation for $20 \mathrm{~min}$ at $4{ }^{\circ} \mathrm{C}$, cells were washed three times and resuspended in PBS. Next, all postvaccination samples were pooled and sorted on a high-speed cell sorter (BD FACSAria III, BD Biosciences at the flow cytometry core facility of LUMC) using an $85 \mu \mathrm{m}$ nozzle and a pressure of 45PSI, while the baseline sample was sorted separately, but in the same collection tube, to ensure an enrichment of plasmablasts/plasma cells (PB/PCs) in the sorted sample. Cells were collected in a BSA-coated Eppendorf tube filled with an RPMI $+10 \%$ FCS solution to avoid excessive cell loss. At baseline, the first 10,000 total CD19+ B cells were sorted, and after adjusting the sorting gate to exclusively sort PB/PCs, another 1201 CD19+CD38++CD24+CD27+ PB/PCs were sorted. For subsequent visits, all available PB/PCs were sorted (11,051 cells). In total, we sorted 22,252 cells. Directly after the sort, samples were spun down and resuspended into the recommended volume (RPMI + 10\% FCS). Further sample processing took place at the Leiden genome technology center (LGTC) in the LUMC according to the 10x Genomics protocols (Figure S1).

\subsection{0x CITE-Seq Transcriptomics and scBCR-Rep Sequencing}

Nearly 22,000 B cells enriched in PB/PCs from different time-points were processed into single cells in a Chromium Controller (10x Genomics) (Figure S1). During this process, individual cells were embedded in Gel Beads-in-Emulsion (GEMs) where all generated cDNAs share a common 10x oligonucleotide barcode. Reverse transcription PCR and library preparation were carried out under the Chromium Single Cell $3^{\prime}$ v3 protocol (10x Genomics) per manufacturer's recommendations. After amplification of the cDNA, a $5^{\prime}$ gene expression library and paired heavy and light chain library were generated from cDNA of the same cell using Chromium Single Cell VDJ reagent kit (scBCR-rep library; v1.1chemistry, 10x Genomics). After library preparation, quality control was performed using a bioanalyzer (Agilent 2100 Bioanalyzer; Agilent Technologies). The libraries were sequenced in the NovaSeq6000 sequencer (Illumina) using the v1.0 sequencing reagent kit (read length: $2 \times 150 \mathrm{bp}$ ). 


\subsection{Unsupervised Clustering of Single-Cell Transcriptomics Data}

The raw sequencing data from different visits were processed using Cell Ranger (v3.1.0) against the GRCh38 human reference genome with default parameters. Single-cell RNA-seq data were processed in R with Seurat (v3.2.3) [9]. Raw UMI count matrices for both expression and hashtags oligos (HTO) generated from the Cell Ranger 10x Genomics pipeline (https://www.10xgenomics.com/; accessed on 27 August 2019) were loaded into a single Seurat object. Cells were discarded if they met any of the following criteria: percentage of mitochondrial counts $>25$; the number of unique features $<100$ or $>5000$. Furthermore, mitochondrial genes, non-protein-coding genes, and genes expressed in fewer than 3 cells were discarded. Moreover, the immunoglobulin $V$ genes were removed from the counts for clustering analysis. The scaled counts of the constant genes were added to the metadata of the Seurat object. We used MULTIseqDemux [10] with default parameters to demultiplex the data to independent visits (corresponding to HTO1-HTO5 tags) and eliminated doublets and negatives among the cells (Figure S2). We found a few T cells ( 20-30 cells) in our data which clustered separately and we removed them from further analysis.

After retaining 5010 singlets, we log normalized the gene counts for each cell using the 'NormalizeData()' function of Seurat with the default parameters. The normalized data were scaled again using 'ScaleData()'. The G2M and S phase scores were regressed out from the Seurat object. Principal component analysis (PCA), selecting the 10 most varying principal components, and t-distributed stochastic neighbor embedding (tSNE) dimension reduction were performed. A nearest-neighbor graph using the 10 dimensions of the PCA reduction was calculated using 'FindNeighbors()', followed by clustering using 'FindClusters()' with a resolution of 0.6. FACS-like plots were generated using transcript average cell scoring implemented in 'TACSplot()' [11].

\subsection{Differential Gene Expression Analysis}

For comparisons between expression values, the Seurat function 'FindMarkers()' was used with the 'Wilcox method'. Cell type markers were obtained using the 'FindAllMarkers()' function with a Wilcoxon signed-rank test. The fold changes of the mean expression level of genes between the selected cell populations were calculated. The pathway analysis of the relevant genes was performed using 'ClueGO' [12].

\subsection{Identification of $V(D) J C$ Genes in the Reconstructed BCRs}

Demultiplexing, gene quantification and BCR contig and clonotype assignment were performed using the Cell Ranger 3.0.2 V(D)J pipeline with GRCh38 as reference. In order to get the BCR of a single cell, we obtained high-confidence contig sequences for each cell and kept the contigs with productive $\mathrm{BCR}$ rearrangements. The assembled filtered BCRs from 6437 cells were submitted to IMGT HighV-Quest for the $V(D) J$ annotation. The constant gene annotation of the BCRs was obtained from the Cell Ranger output. The usage of constant gene in this individual was identified from the single-cell data and was compared with the constant gene usage identified by the flow cytometry. All chains (single, pairs, threes or fours) were retained. Of 6437 cells with BCRs, 4051 single cells had one heavy chain recorded in the Seurat object. The repertoire analysis was performed on both the sets (6437 and 4051 cells) and the discussed set is implicitly mentioned in the text or figure legends. In case two or more in-frame/productive heavy chains were recorded from one cell, both the chains were included in the repertoire analysis.

\subsection{Identification of B-Cell Clonotypes}

Multiple files obtained from the IMGT HighV-Quest were combined using an in-house Python script. The B cell clonotypes were identified with the same $V$ family, $J$ gene, and $80 \%$ nucleotide identity in IG heavy chain CDR3 as computed by Levenshtein distance \{Python\}. As $V$ gene assignment might be flawed by the assignment methods, we used the $V$ family to call clonotypes which avoids inaccurate grouping of the clones in the first place. 
Also, a comparative assessment was performed of the clonotypes obtained when using $V$ genes versus $V$ family in assigning B cell clonotypes.

\subsection{Analysis of scBCR-Rep and Clonotypes}

The Circos plots of $V J$ usage were made using circlize $\{R\}$. To plot identified clonotypes, IGHC usage, class-switching events and CDR3 length usage, ggplot $2\{R\}$ was used. We used bcRep $\{R\}[13]$ to plot the amino acid usage in the amino acid sequence of the junction. BCellMA $\{R\}[14]$ was used to plot the replacement amino acid mutations from IMGT HighV-Quest output tables. Clonal lineages were built using alakazam [15] and plotted using igraph $\{R\}$. The clonal lineages were built based on the SHMs introduced in the $V$ gene sequence. The $V$ genes were compared to the germline $V$ gene in the IMGT database and the number of mutations in the clonal group was used to build these lineages. In case two different mutation lineages were detected in a clonal group and an intermediate BCR was missing, the tool alakazam incorporated an inferred node suggesting a missing BCR that could not have been sampled and/or sequenced.

\subsection{Query Tool to Identify Anti-Toxoid BCRs}

The currently profiled BCRs comprise of all the $\mathrm{PB} / \mathrm{PC}$ raised in response to the vaccine which comprises of multiple components. To identify the contribution of each component of the vaccine, we developed an in-house tool using Python. To show its functionality, junction sequences related to anti-TT, anti-DT, anti-FHA, anti-Prn and anti-PT were searched in the literature. We could only identify the CDR3 sequences for anti-TT, antiDT and anti-PT. These sequences were searched in the Boostrix-specific BCR-rep obtained in this study. The search was not only performed based on the percentage identity, but a parameter of length flexibility was also incorporated; i.e., the search space will include junction sequences that are longer or shorter by a user defined length. The sequence similarity is calculated based on the Levenshtein distance of the junction sequences.

\section{Results}

\subsection{Single-Cell Transcriptome Landscape of the Cells from a Vaccinated Individual}

3.1.1. Majority of the PB/PCs Derived from d5 and d7 Post-Vaccination

We measured the transcriptomics and BCR-rep data at the single-cell level from the longitudinal samples obtained from an individual following Boostrix vaccination. After applying quality checks to the transcriptomics data from 10,000 total CD19+ B cells and 12,252 CD19+CD38++CD24+CD27+ PB /PCs from five pre-defined time-points (Figure S1), we obtained 5010 singlets (Figure S2). Unsupervised clustering using Seurat distinguished seven different cell clusters based on their transcription profile (Figure 1A). CD19, CD38, CD24 and CD27 markers used to define the cells by flow cytometry allowed discrimination between B cells (Figure 1A; Clusters B1-B3) and PB/PCs (Figure 1A; Clusters P1-P4). Based on the tag mapping we could identify the sampling time-point of individual cells. The highest number of cells was obtained from $\mathrm{d} 0$ when both total $\mathrm{B}$ cell and PB/PCs were sorted. At follow-up visits, more PB/PCs were obtained at $\mathrm{d} 5$ and $\mathrm{d} 7$ and clearly less at d10 and d14 (Figures 1B and S2B). This reflected the number of sorted cells (Figure S1B), but mostly post-vaccination fluctuations in the number of PB/PCs (Figure S1C), which were also observed in the flow cytometry data obtained from this donor previously [5]. 
A

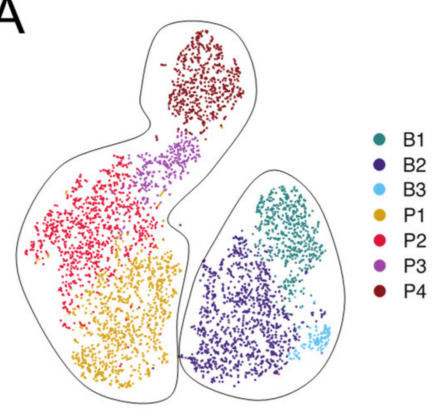

B

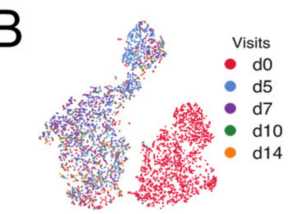

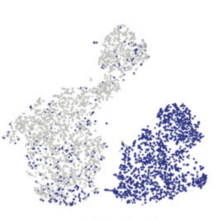

d0: 2222 cells B cells: 1878 cells
PB/PCs: 344 cells

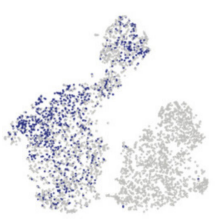

d7: 1004 cells

C

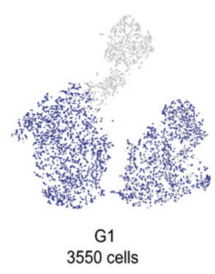

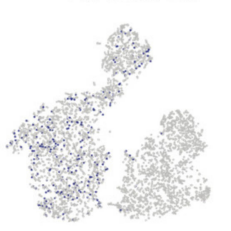

d10: 334 cells

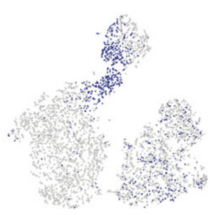

$\mathrm{S}$
855 cells

Cell cycle phase
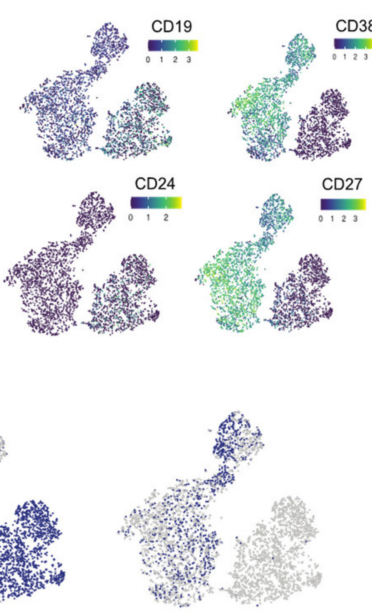

d5: 1086 cells

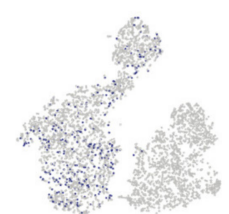

d14: 364 cells

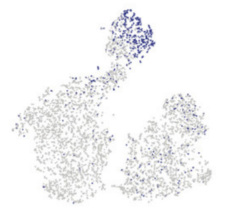

G2
605 cells

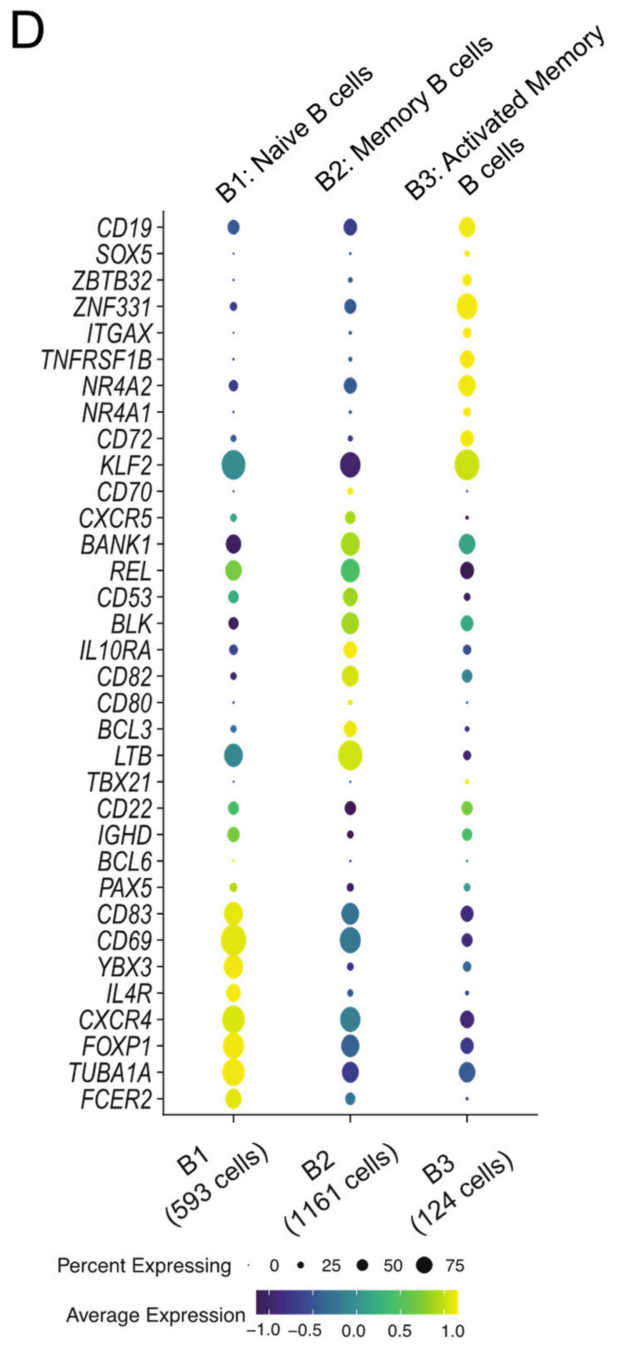

Figure 1. Identification of B-cell subpopulations by single cell-transcriptomics analysis. (A) The clusters of cells are represented on a tSNE plot based on the highly variable genes. The clusters are named as B1-B3 for B cells and P1-P4 for PB/PCs. The expression of the markers used for sorting the cells in the study is plotted on the tSNE plot. High expression of CD38 and CD27 marks the clusters P1-P4 as the PB/PCs. (B) Plotting tag information on the tSNE plot. The cells are assigned to each visit and cells at $\mathrm{d} 0$ are assigned to B cells and PB/PCs based on their location in B1-B3 and P1-P4 clusters, respectively. (C) Visualizing cell cycle scores on tSNE plot. G1, S and G2M cell-cycle scores are represented on the tSNE plot where P3 and P4 clusters comprise of S and G2M phase cells. (D) Dot plot of the gene markers used for identifying the B-cell subpopulations. B1: Naive B cells; B2: Memory B cells; B3: Activated memory B cells.

\subsubsection{Proliferating Cells Are Clustered in One Cluster}

Despite having regressed out cell cycle influence, the P4 cluster separated based on the expression of the cell cycle genes (Figure 1C). A majority of the cells in all the clusters, except P3 and P4, were in G1 with a few cells in S phase, whereas P3 and P4 clusters were heavily comprised of the cells belonging to $S$ and G2M phase (Figure 1C), suggesting that most proliferating cells are clustered in P4. The cells present in G1 phase are the mature PB/PCs deemed to produce Abs which are mostly present in P1 and P2 clusters.

3.1.3. B1-B3 Clusters Correspond to Naive, Memory and Activated Memory B Cells, Respectively

The B-cell clusters i.e., B1-B3 were assigned individual identities based on the differentially expressed genes among these clusters and the classical markers and/or literature. 
The B1 cluster expressed classical markers of naive B cells i.e., IGHD, CXCR $4^{\text {high }}$ [16] and FOXP1 [17] (Figure 1D). Cluster B2 was defined as memory B cells based on overexpressed of unique markers e.g., $B A N K 1, R E L$. The genes $B C L 3$ and $L T B$, that promote $A b$ production, were also highly expressed in cluster B2 [18]. The SOX5, ZBTB32 are markers for activated memory B cells which were also representative markers for cluster B3, hence the 124 cells in cluster B3 were identified as activated memory B cells [19]. The decreasing expression of CXCR4 marker from naive to memory to activated memory $\mathrm{B}$ cells was observed in clusters $\mathrm{B} 1$ to $\mathrm{B} 3$, which was also mentioned in the similar subsets identified from an influenza vaccination study [19]. Overall, we could discriminate between naive B cells (cluster B1), memory B cells (cluster B2), activated memory B cells (cluster B3) and PB/PCs (clusters P1-P4) based on markers unique to these cell types (Figure 1A,D).

\subsection{Flow Cytometry Guided Plasma Cell Maturation in the Single-Cell Transcriptome 3.2.1. Staging of $\mathrm{PB} / \mathrm{PC}$ s Based on Flow Cytometry Markers}

Previously, we used longitudinal flow cytometry data from 10 vaccinated donors to establish how expression of selected cell surface markers changes during maturation of PB/PCs [5]. To visualize the plasma cell maturation in the here studied donor, the $\mathrm{PB} / \mathrm{PCs}$ data from days $0,5,7,10$ and 14 were merged in the Infinicyt software (Infinicyt ${ }^{\mathrm{TM}}$ Software v2.0, Cytognos) and the maturation was defined by the downregulation of CD20 and upregulation of CD138 (Figure 2A left panel). The most mature cells exhibited CD20CD138+ phenotype. Then we traced changes in expression of four additional markers i.e., CD19, CD27, CD62L, and CD38. Based on the expression of all six markers, we drew new maturation pathways and divided it into six continuous maturation stages (Figure 2A right panel). Similar to flow cytometry staging, after removing B cells, $\mathrm{PB} / \mathrm{PC}$ s were clustered based on all the genes and arranged with phenotype ranging from CD20+CD138- (less mature) to CD20-CD138+ (most mature) (Figure 2B). However, the cells from different clusters were mixed and the maturation based on P1-P6 clusters could not be drawn. Therefore, we clustered the PB/PCs again based on the expression of only 6 genes (SELL (CD62L), TNFRSF7 (CD27), SDC1 (CD138), ADPRC1 (CD38), MS4A1 (CD20) and B4 (CD19)) and used the expression of $\mathrm{CD} 20$ and $\mathrm{CD} 138$ to align the clusters with the maturation pathway of the PB/PCs. Herein, based on the maturation pathway defined by CD20 and $\mathrm{CD} 138$ markers, a trajectory from the blue-colored clusters (Pa1) to the dark red ones (Pa10) can be drawn (Figure 2C). Based on the maturation of clustered cells the staging of the $\mathrm{PB} / \mathrm{PCs}$ was performed from Pa1-Pa10 and the expression of other markers was assessed (Figure 2D). 
A
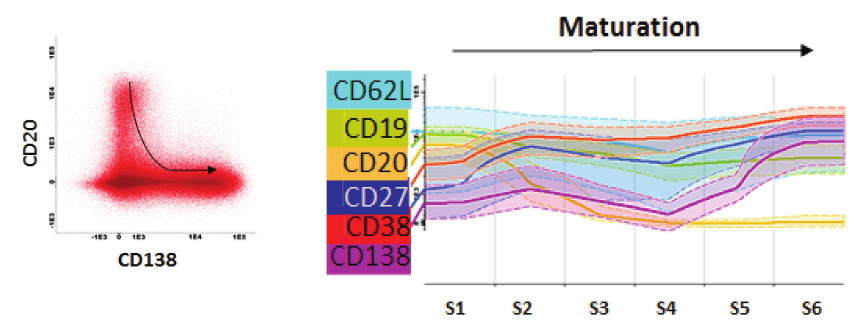

C

E
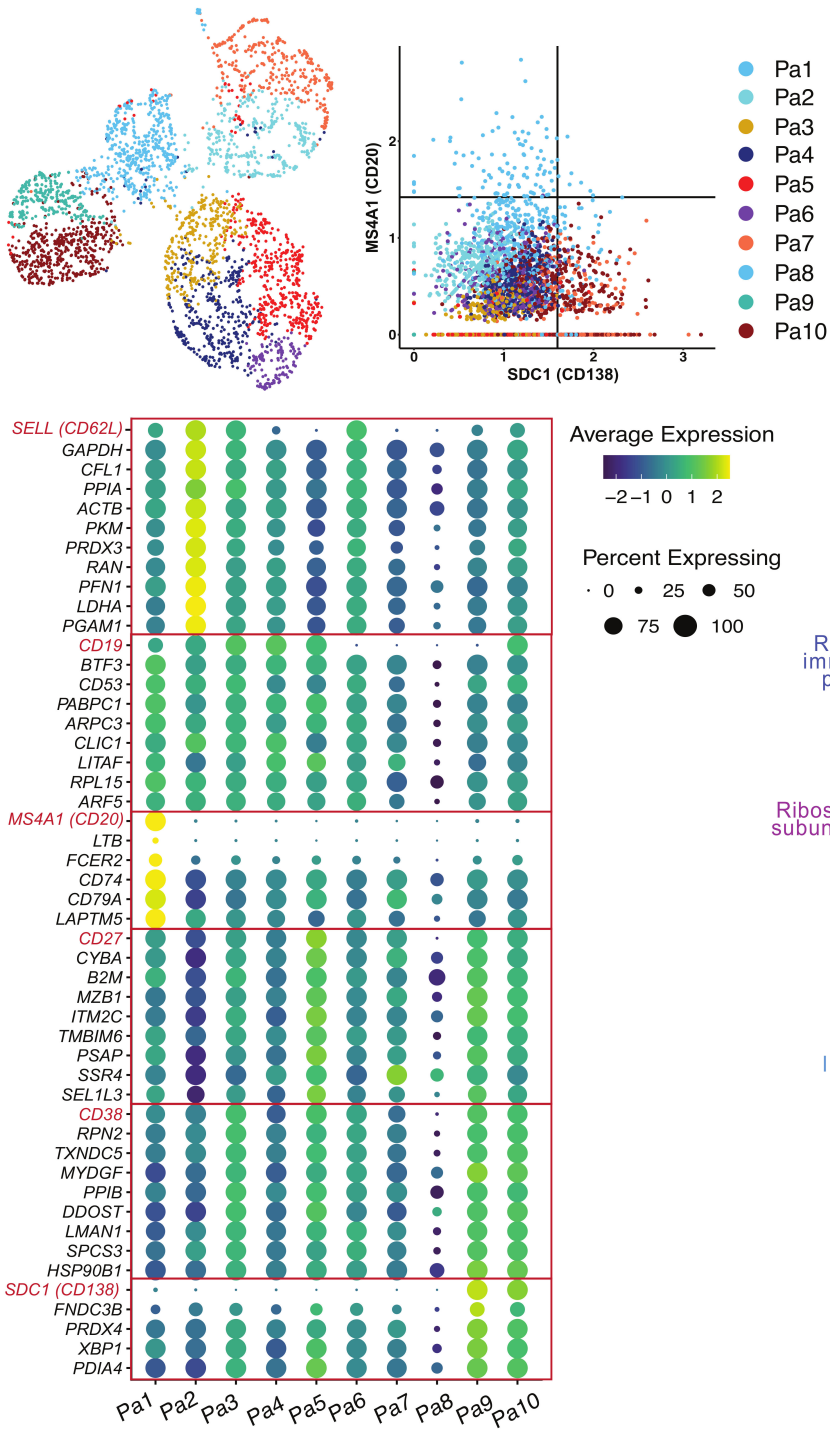

B
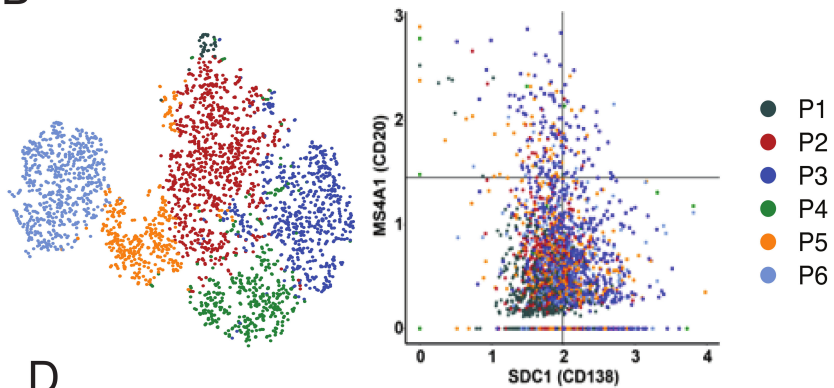

$\mathrm{D}$

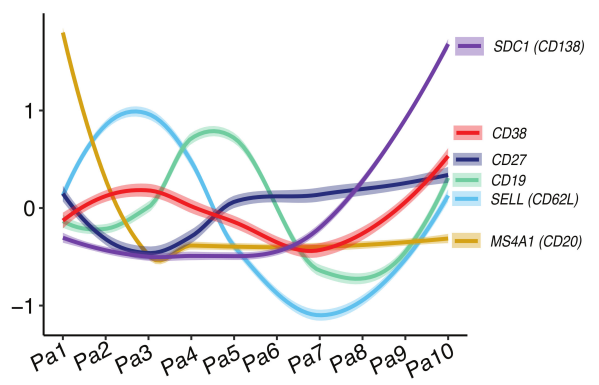

$\mathrm{F}$

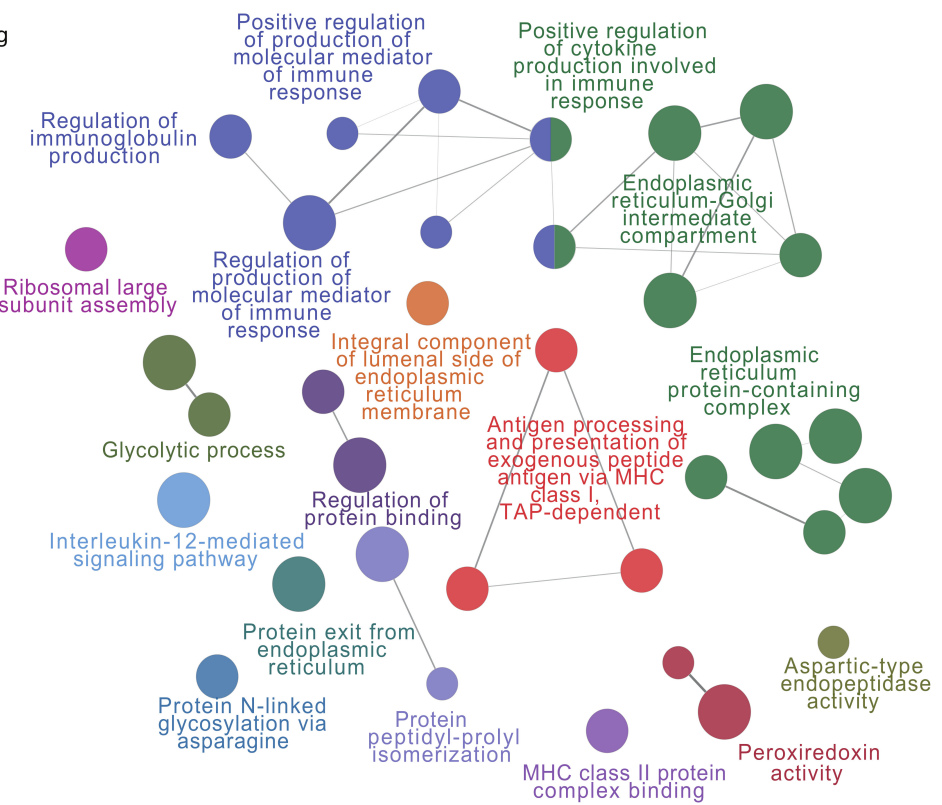

Figure 2. Plasmablast/plasma cell maturation using flow cytometry and single-cell transcriptomics data over time following vaccination. (A) Flow cytometric evaluation of cell surface markers in maturing PB/PCs. Plasma cell maturation pathway was drawn in the Infinicyt software based on the surface expression of CD20 and CD138. The least mature PB/PCs were defined as CD20+CD138- and the most mature as CD20-CD138+. Dot plot in the left panel shows all PB/PCs from the same donor and time-points used in the sequencing, and black arrow indicates the direction of plasma cell maturation Each dot represents one cell. Subsequently, the entire maturation pathway was divided into 10 maturation stages (S1-S6), for which expression of additional cell surface markers was evaluated (right panel). (B) FACS-like plot to arrange PB/PCs clusters P1-P6 in the maturation pathway defined by the expression of CD20 and CD138. (C) Redefining stages of PB/PCs in single-cell transcriptome data using six markers pre-selected based on flow cytometry. The PB/PCs were clustered using six pre-defined maturation markers and FACS-like plotting is used to stage the plasma cell clusters based on the maturation pathway. Ten clusters i.e., Pa1-Pa10 were obtained. (D) The spaghetti plot of all six markers visualized after staging the 
PB/PCs. (E) Additional maturation markers identified using single-cell transcriptomics data based on the Pa1-Pa10 plasma cell clusters and corresponding stages. Genes following similar expression patterns were identified using the TACSplot function. (F) The important pathways identified based on the top 20 similar genes to each of the six maturation genes. The GO terms based on biological processes, molecular pathways, cellular components and immunological processes were merged. The pathways with $<0.05$ corrected $p$ values were selected for visualization.

\subsubsection{Pathways and Additional Membrane Markers Identified in Relation} to the Maturation of PB/PCs

Based on the 10 clusters/stages of the plasma cell maturation, we identified additional markers that might be changing during the maturation of the PB/PCs (Figure 2E). Genes with similar expression to the six maturation markers were selected from the single-cell transcriptomics data (Table S1; Figure 2E). The HLA locus-specific genes were not included in the analysis. Several of these genes are involved in the regulation of immunoglobulin production, molecular mediation of immune responses, $N$-glycosylation, antigen processing and presentation, protein folding and cytokine production (Figure $2 \mathrm{~F}$ ). The majority of these pathways directly suggest the role of these genes in the Ig secretion, post-translational modifications to ensure correct folding of the proteins and henceforth an optimum response of the immune system to the vaccines. This is in line with the preparation for immunoglobulin secretion, which is the major role of most mature PB/PCs.

\subsection{Characteristics of $V(D) J$ Usage over Time upon Vaccination}

\subsubsection{Identification of Ig Heavy and Light Chains in Majority of the Sequenced Cells}

Additional to transcriptomics profiling, we measured the BCRs of the cells from the longitudinal samples obtained from an individual after Boostrix vaccination. The barcode mapped BCR information for each cell was obtained from the Cell Ranger output. We filtered out the unproductive rearrangements and could map at least one heavy or light chain for 4175 cells (Figure S3A). No chain was recorded for 835 cells (of which 818 cells were collected at d0) (Figure S3A), most likely owing to the low expression of BCR in non-plasma cells. We also observed that the cells comprised multiple chains varying from three to five heavy and/or light chains ( $~ 8 \%)$. Therefore, we filtered out additional chains that were supported by a low number of reads as compared to the other chains from the same cell. In case multiple productive chains were supported by similar number of reads, we included them all in our analysis. After filtering, we found varying chain numbers $(1,3$ or 4 chains) mostly for B cells identified at d0 (Figure S3A), which partly could be related to the low expression of BCR or possible doublets. The BCRs with 3 or 4 chains comprised of completely different $V, D, J$ and $C$ genes for both heavy and light chains, which might be related to the doublets that could not be removed in the filtering steps or the production of multiple chains in B cells [20]. For further comparisons in the manuscript, the BCRs from $\mathrm{d} 0 \mathrm{~B}$ cells and $\mathrm{PB} / \mathrm{PC}$ were considered independently and post-vaccination BCRs were compared with d0 PB/PCs. Also, the expression of BCRs in B cells for both heavy and light chains was lower as compared to plasma cells.

\subsubsection{Cells with Different IGHV and IGHJ Are Dominant at Different Time Points}

The single cells with paired heavy and light chain information revealed preferential pairing between IGHV genes and IGKV1 and IGKV3 gene families (Figure S3B). IGLV genes mostly belonged to the IGLV1, IGLV2 and IGLV3 gene families. Also, over time, IGKV1, IGKV3, IGLV1, IGLV2 and IGLV3 gene families remained the most abundant, with minor variations in the usage which can be due to the limited number of cells or expansion of individual clones. However, no strong selection for a particular light chain was observed. On the contrary, we did observe changes in the usage of the IGHV genes over time (Figure S3B). Overall, the IGHV3 was the most used gene family, however, the usage of IGHV1, IGHV2, IGHV4 gene families increased in PB/PCs collected at d5 and $\mathrm{d} 7$ (around the peak of plasma cell response). To better understand the dynamics of the gene usage in IGH chains, we looked into the $V(D) J$ gene usage. We found that IGHD3 
genes were used the most over all time-points, whereas the usage of IGHV and IGHJ genes changed over time (Figure S3C). We observed that the increased usage of IGHV1, IGHV2, IGHV4 gene families was accompanied by an increased usage of IGHJ2, IGHJ3 and IGHJ6 genes post-vaccination.

\subsubsection{IGHV3 and IGHJ4 Genes Predominant in $\mathrm{d} 0 \mathrm{~PB} / \mathrm{PCs}$}

As we observed increased usage of several IGHV and IGHJ gene families, we examined the usage of IGHV and IGHJ gene families in all cells across different visits at baseline and post-vaccination. We observed more restricted scBCR-rep in $\mathrm{d} 0 \mathrm{~PB} / \mathrm{PC}$ as compared to d0 $\mathrm{B}$ cells (Figure S3D). While 55 unique of $158 \mathrm{VJ}$ pairs were observed in d0 B cells, only 16 unique usage of $118 \mathrm{VJ}$ pairs were present in $\mathrm{d} 0 \mathrm{~PB} / \mathrm{PCs}$. The IGHV3-23 and IGHJ4 were the most used pairs in both $\mathrm{d} 0 \mathrm{~B}$ cells and $\mathrm{PB} / \mathrm{PCs}$. The majority of the recombination events observed in d0 B cells used IGHV1, IGHV3, IGHV4, IGHV5 families recombining with IGHJ4, however, it was highly selective in d0 PB/PCs, i.e., the IGHV3 family genes and IGHJ4 gene were used the most.

\subsubsection{Few Clonotypes Expand with Delay at $\mathrm{d} 10$}

While comparing the PB/PCs from post-vaccination visits to $\mathrm{d} 0 \mathrm{~PB} / \mathrm{PCs}$, we observed that the overall diversity increased at all the time-points post-vaccination (Figure 3A). We observed 50 and 65 new $V J$ pairs at $\mathrm{d} 5$ and $\mathrm{d} 7$ post-vaccination, respectively. Despite comparable numbers of sorted PBMCs and plasma cell counts at d10 and d14, we observed 24 new VJ pairs at d10 post-vaccination as compared to 35 new VJ pairs used at d14. Overall, we observed high usage of the IGHV3-23 gene and the IGHV4 gene family at all time-points including baseline. A clear expansion of IGHV1 and IGHV4 family genes especially IGHV1-2, IGHV1-18, IGHV3-33, IGHV4-39, IGHV4-31 gene can be observed around the peak of plasma cell expansion (Figure 3A; d5, d7 and d10). IGHV5-51 usage was also observed to be expanding at $\mathrm{d} 10$. The expansion of selected few $V(D) J$ genes at the later time-points suggested the expansion of few clones with a delay. When comparing the relative usage of IGHV and IGHJ genes between visits post-vaccination, we observed that the usage of majority of the genes is comparable between visits with few exceptions, for example, the usage of IGHV1-69 increased at $\mathrm{d} 5$ and $\mathrm{d} 7$ post-vaccination (Figure 3B). Furthermore, IGHV1-2 and IGHJ3 were used the most at d5, whereas IGHV1-2 and IGHJ4 were used at $\mathrm{d} 7$, while at $\mathrm{d} 10$ and $\mathrm{d} 14$ the usage of IGHV1-2 was minimal.

A

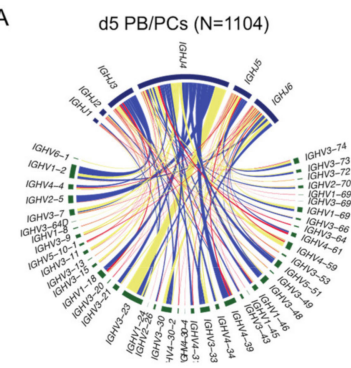

7 PB/PCs (N=1110)

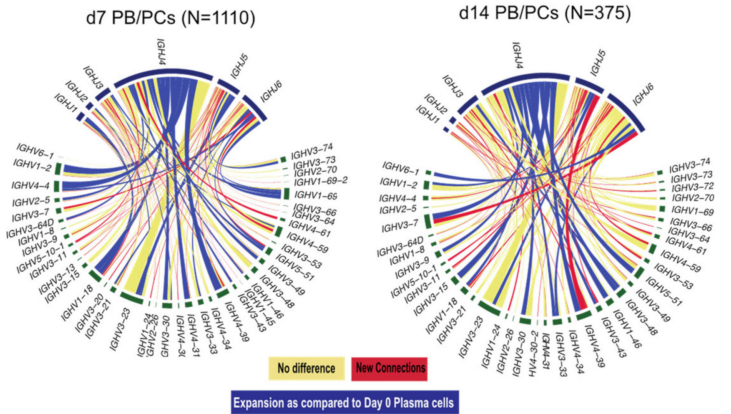

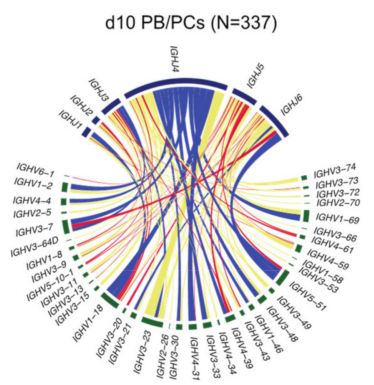

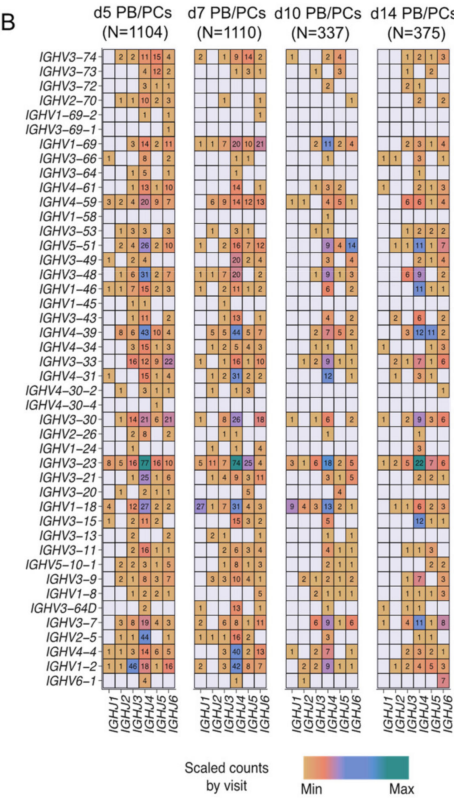

Figure 3. VJ gene usage in $\mathrm{PB} / \mathrm{PC}$ from all the post-vaccination visits relative to baseline. (A) Circos plot of VJ gene usage 
in PB/PCs from all the post-vaccination visits relative to baseline. Each ribbon represents a specific pairing between $V$ and $J$ genes, and the ribbon width represents the confidence bounds of the plotted data using the LOESS method. Ribbons color shows no change (yellow) or expanded links (dark blue) or new recombination events (red) as compared to d0 PB/PCs. (B) Heatmap plot of VJ gene usage in PB/PCs from all the post-vaccination visits.

\subsection{Constant Gene Usage over Time upon Vaccination IGHG1 Constant Gene Predominant at the Peak of the PB/PCs}

Assessment of the constant gene usage helps in understanding the raised effector function post-vaccination. To identify the usage of the constant genes in the profiled BCRs, we grouped the receptors based on the constant genes. IGHM was the most used constant chain in d0 B cells (Figure 4A), followed by IGHD. It was in line with the abundance of naive/pre-germinal center B cells in the pre-vaccination scBCR-rep. However, IGHA1 was the most used constant gene in the $\mathrm{d} 0 \mathrm{~PB} / \mathrm{PC}$ (Figure 4A). The composition of the constant chains changed after vaccination and IGHGI became the most used chain. The percentage of cells utilizing IGHA1 decreased at the peak of the plasma cell response i.e., $\mathrm{d} 5$ and $\mathrm{d} 7$, but at d10 and d14 returned to the levels comparable to baseline. The usage of IGHD was negligible in the PB/PCs post-vaccination. On the other hand, IGHM usage reduced at the peak of PB/PCs response which returned to original levels at d14. We observed a similar trend in the flowcytometry data, wherein the $67 \%$ of PB/PCs expanded at d7 was IgG1+ and the relative usage of IgA1 was reduced (Figure 4B). The IGHD expression was relatively low in the single-cell data limiting identification of the $\operatorname{Ig} M+\operatorname{IgD}+\mathrm{B}$ cells, which we could indeed identify in the flow cytometry data (data not shown). With the paired chain information from single cells, we observed that the IGKC gene was the most used gene followed by IGLC2 in all the post-vaccination visits. The high usage of the IGKC gene is in line with our previous results specifying high usage of IGKV gene families (Figure 4C). Interestingly, in d0 PB/PCs, IGLC1, IGLC2 and IGLC3 genes were used more frequently than other IGLC genes.

\subsection{BCR Clonotypes over Time upon Vaccination}

\subsubsection{Identification of Clonotypes Based on $V$ Family Grouping}

The BCRs were clustered into clones/clonotypes with a slight deviation from the classical definition of clonotypes; instead of genes, we used $V$ family and $J$ gene for grouping. Irrespective of the definition used, results of the clustering were highly similar (rand index of 0.99). The differences in the number of clusters were caused by the fact that several singletons clustered with other singletons with similar BCRs when the $V$ gene definition was relaxed by $V$ family (Table S2). Of 3334 clonotypes, 662 clonotypes could not be assigned any visit, 575 of which were singletons ( 8 clones with $>10$ BCRs; the largest clone comprises 129 BCRs). Overall, 936 of 3334 clonotypes had at least 2 cells. 369 of 936 clonotypes were shared between at least two visits of which 145 clonotypes had cells present on $\mathrm{d} 0$. For the 4051 cells derived from the known visits and with a rearranged productive heavy chain, Figure 5A shows the origin of the top 30 (449 BCRs) of 145 (2075 BCRs) clonotypes. The largest clone comprised of $46 \mathrm{~B}$ cells and was present in all visits but d14 (Figure 5A; C115). However, 30 additional B cells belonged to the same clonotype, but could not be assigned any visit (Figure S4A; the top second clone). The clonotype C115, consisting of only the IGHG1 isotype, had an expansion of identical sequences across multiple visits including baseline as visualized by the largest pie in Figure 5B. This largest node comprises of 58 individual BCR sequences wherein 34 of them are derived from $\mathrm{d} 5$ and $20 \mathrm{BCR}$ could not be assigned to any visit. 
A

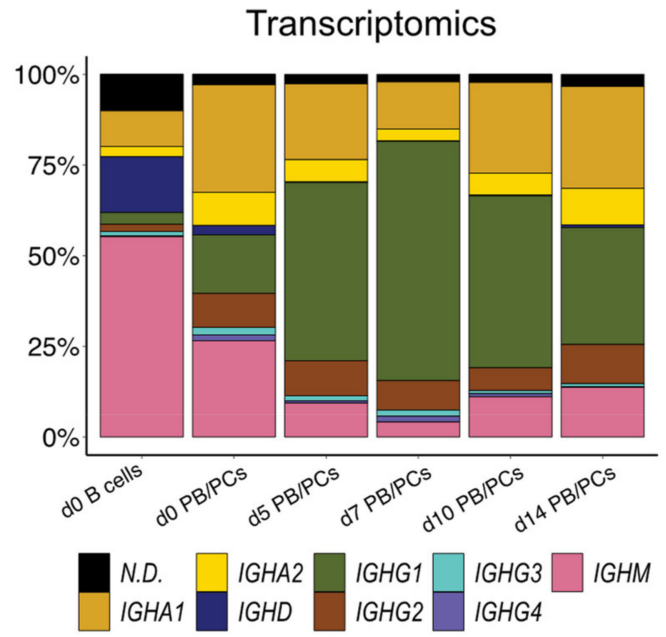

B

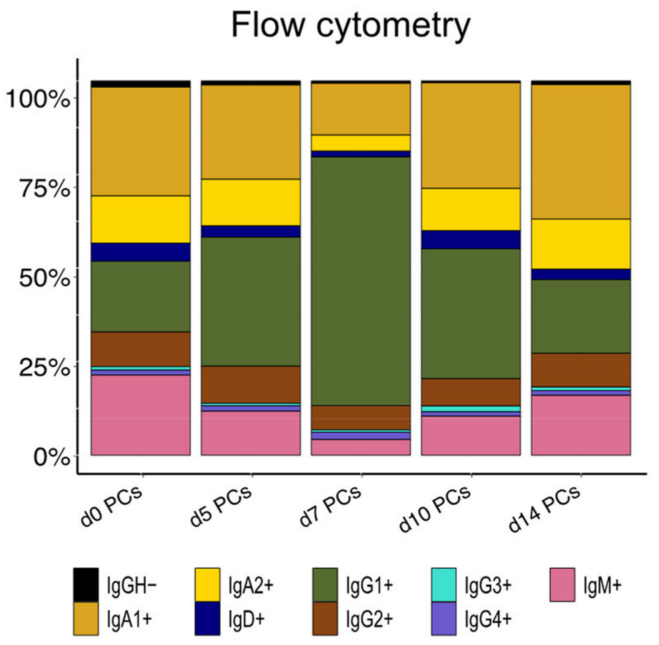

C
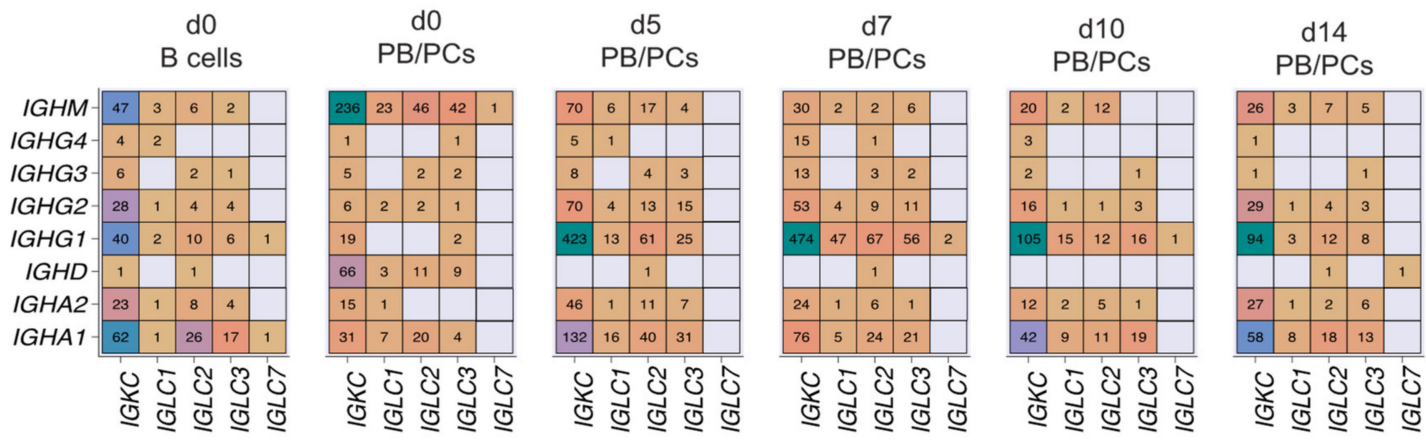

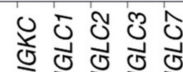

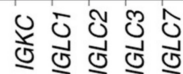

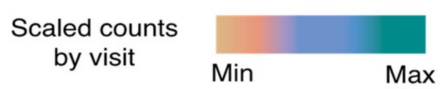

Figure 4. The dynamics of heavy and light constant gene usage in the BCRs over different time-points pre- and postvaccination. (A) Usage of IGH constant genes in single-cell BCRs over time. The PB/PCs and B cells at the d0 are considered separately. (B) Over time usage of constant genes in the PB/PCs as identified by flow cytometry (based on membrane and intracellular Ig expression). (C) The usage of heavy and light constant genes across different visits. The numbers in the heatmap are the number of chains using a given pair and the colors are ranged based on the scaled counts for each visit.

\subsubsection{CSR Events within the Clonotypes}

We investigated the clonotypes for the CSR events by querying clones with different IGHC chains. Of 369 clonotypes shared between different visits, 191 clonotypes used the same constant gene and do not represent clonotypes with CSR. Interestingly, a majority of clonotypes (53\%; 101 of 191) only used the IGHG1 gene, 21\% clonotypes used IGHA1 followed by $10 \%$ using IGHM and $8 \%$ using IGHG2. Thus, clonotypes followed the same trend as constant gene usage. Of 178 clonotypes that used multiple constant genes, $17 \%$ used IGHG1 and IGHA1 (Figure 5C). IGHG1 is clustered with IGHM three times more (74\%) as compared to IGHG2 clustering with IGHM. Similarly, IGHA1 is clustered with IGHM three times more than IGHA2, suggesting high CSR between IGHM and IGHA1. Overall, we found that $45 \%$ of these clonotypes contained IGHM and IGHG isotypes, followed by IGHM and IGHA (29\%). The usage of multiple constant genes in the clonotypes suggest a high IGHG1 response followed by IGHA1. 


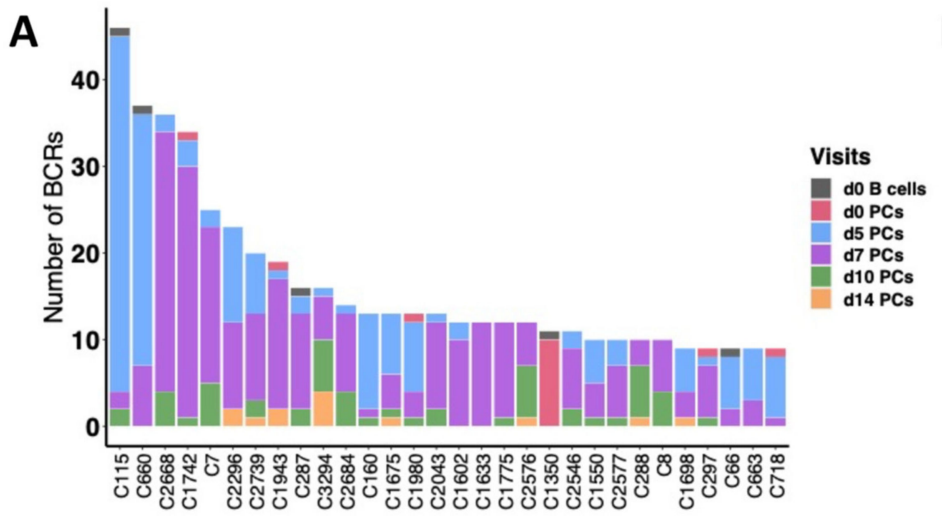

C

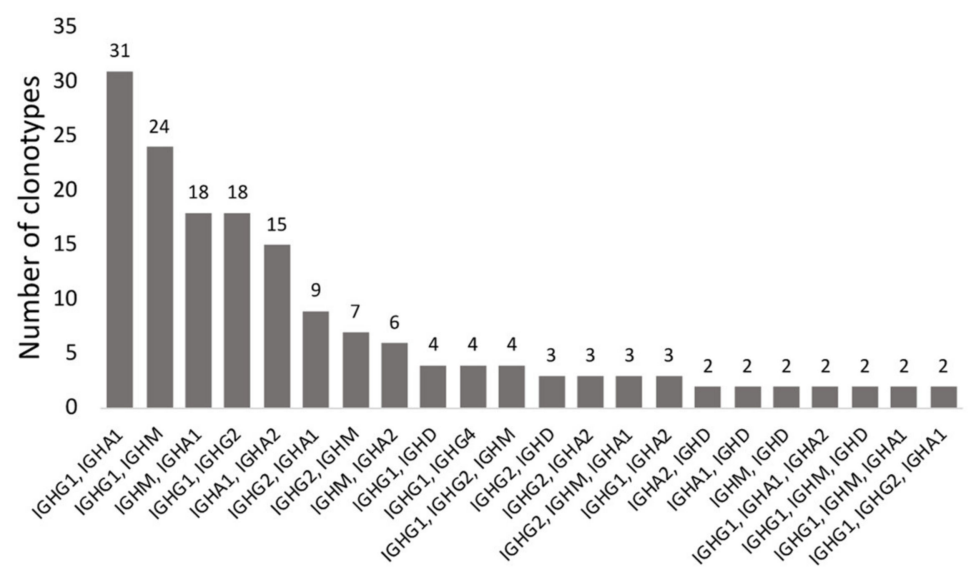

B

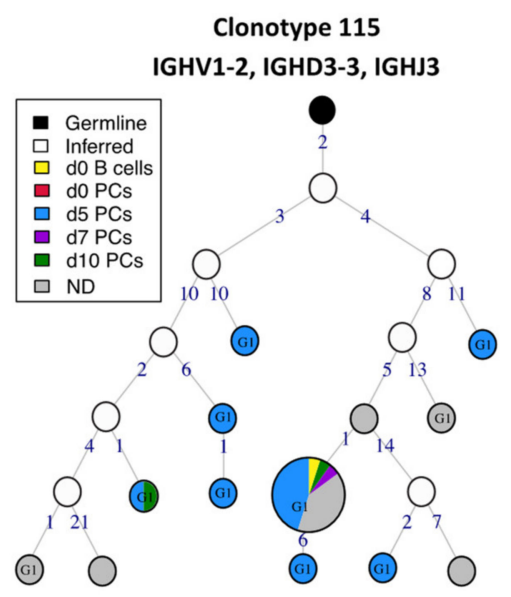

D

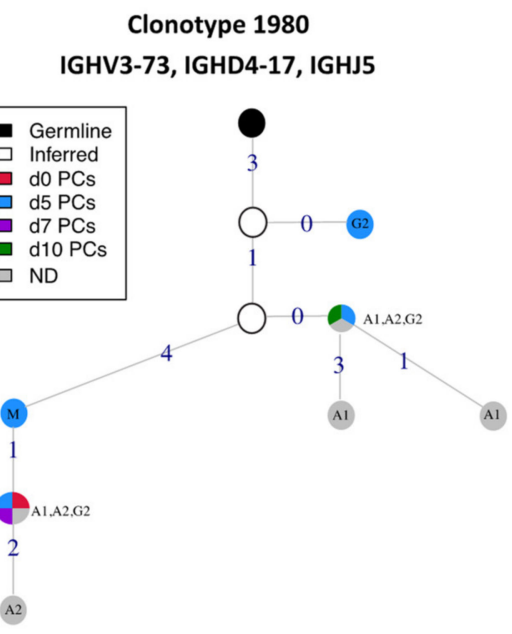

Figure 5. BCR clonotypes over different time-points. (A) 30 most numerous clonotypes over different time-points preand post-vaccination. Contribution of different visits to the total size of a clone is indicated by colors. (B) Clonal lineage of clonotype 115. The graph is constructed using $V$ gene germline sequence as the root node. The size of each node is scaled and directly related to the number of BCRs for that particular sequence. The nodes are colored based on the visits from which sequences are derived. The unique sequences present across multiple visits are also grouped and colored differently as mentioned in the legend. Germline is colored black and the inferred nodes are colored white. The edge label indicates the number of different nucleotides as compared to the previous node. (C) A bar plot indicating the $>2$ clonotypes containing cells with multiple constant genes. For example; each of 31 clonotypes consist of sequences using IGHG1 and IGHA1 constant genes. The number of clonotypes for each combination is mentioned on the top of bar. (D) Clonal lineage of clonotype 1980 with class-switch recombination events. Additional inferred nodes were added to correctly represent the order of class-switching.

\subsubsection{Clonal Lineages of the Clonotypes with CSR Events}

We selected a few of the large clonotypes to visualize the class-switching process via lineage construction. While some of the clones (e.g., clone 660, utilizing almost exclusively IGHG1 with one IGHA1) were highly mutated and diverse (Figure S4B), others (e.g., clone 1980) showed a more conserved mutation pattern with very few mutations separating the clone members (Figure 5D). Clone 1980 is the 20th largest clone where the IGHA and IGHG constant genes expanded with identical sequence in the $V$ genes, which were only one to four mutations away from the IGHM harboring BCR (Figure 5D). This is an example of a receptor wherein the $\mathrm{BCRs}$ at later time-points harbor fewer mutations as compared to the germline than the BCRs sampled on $\mathrm{d} 0$. This suggests that this clone is derived from memory responses generated during previous antigen encounters. 


\subsubsection{Comparable Length of Most of the Junctions}

Similarly, CDR3 length distribution was plotted for all the cells grouped by clonotype at baseline and each visit post-vaccination. We observed a larger proportion of clonotypes with CDR3 lengths of 12 amino acids at $\mathrm{d} 5$ (mean length of CDR3: $15.8 \pm 3.8$ amino acid residues) and 15 amino acids at $\mathrm{d} 7$ (mean length of CDR3: $15.6 \pm 3.1$ amino acid residues). We also observed a few clonotypes with longer junction region at $\mathrm{d} 5$ and $\mathrm{d} 7$ post-vaccination (Figure S4C).

\subsection{Mutation Load in BCRs upon Vaccination}

The selection process for higher affinity Abs during the GC response also leaves an imprint on the $I G H V$ genes, in terms of the type of amino acid substitutions observed in the cells which have successfully passed selection. Analysis of such mutation patterns at the nucleotide level allows us to understand the adaptability of the immune system to the antigens encountered during vaccination.

\subsubsection{Positively Correlated Mutations in Heavy and Light Chain Genes}

The majority of the BCRs in the activated memory B cells used the IGHM (34 cells) gene followed by the IGHA1 (6 cells) constant genes. As expected, the mutation load between memory B cells (cluster B2) and activated memory B cells (cluster B3) was not significantly different. We observed that the IGHG1 and IGHM BCRs in PB/PCs had significantly more mutations $(p<0.05 ;<0.001)$ in the complete $V$ gene as compared to the memory and activated memory B cells sampled at $\mathrm{d} 0$ (Figure 6A). The mutations in $V$ region in sequences using IGHG1 constant gene are also accounted for in the CDR1 and FR3 region whereas for IGHM the significant differences were also observed in FR2, CDR2 and FR3 regions. We observed that IGHV of BCRs utilizing IGHG1, in general, harbored more mutations in post-vaccination visits as compared to other constant genes in all $V$ regions (Figure 6A), which might be associated with the expansion in the IGHG1 BCRs post-vaccination. Moreover, we found that the mutations in heavy chains were positively correlated with the mutations in the light chain in all the regions with significant $p$ values (Figure 6B).

\subsubsection{Positive Selection of Mutations in the CDR Region over Time}

To further confirm that the mutations in PB/PCs were antigen driven, we employed BASELINe [21] to detect the selection by analyzing mutation patterns in experimentallyderived Ig sequences. We observed a negative/neutral selection in framework regions and slightly positive selection in the CDRs (Figure S5). The differences between the selection estimates in both FWR and CDR regions were highly significant $(p \leq 0.03)$ except in FWR region at visit $\mathrm{d} 10(p=0.25)$. The positive selection of mutations in CDRs and negative selection in FWRs correspond with normal antigen-specific B cells [22]. Moreover, we did not observe a varying trend i.e., increasing or decreasing selection pressure in CDRs over time suggesting that the selected receptors were already available at the beginning of the response and these were recruited over time for optimum immune response. 
A

\section{IGHA1}

$$
\text { IGHA2 }
$$

IGHG1

IGHG2

IGHG3

IGHG4

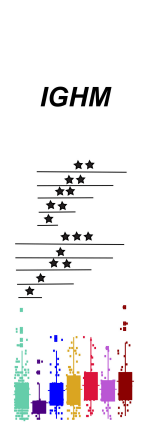

\section{FR1 REGION}

VREGION

\section{CDR1 REGION}
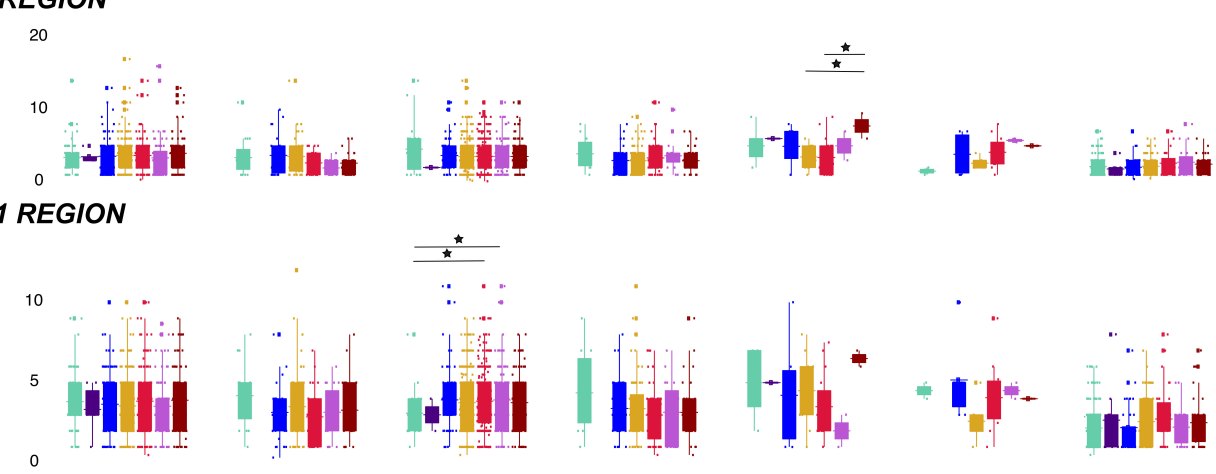

FR2 REGION
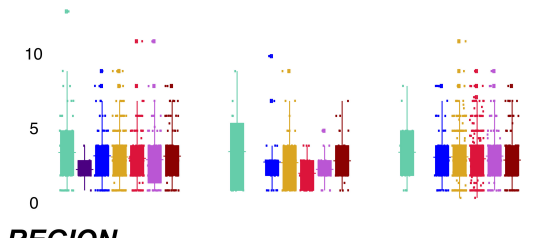

\section{CDR2 REGION}
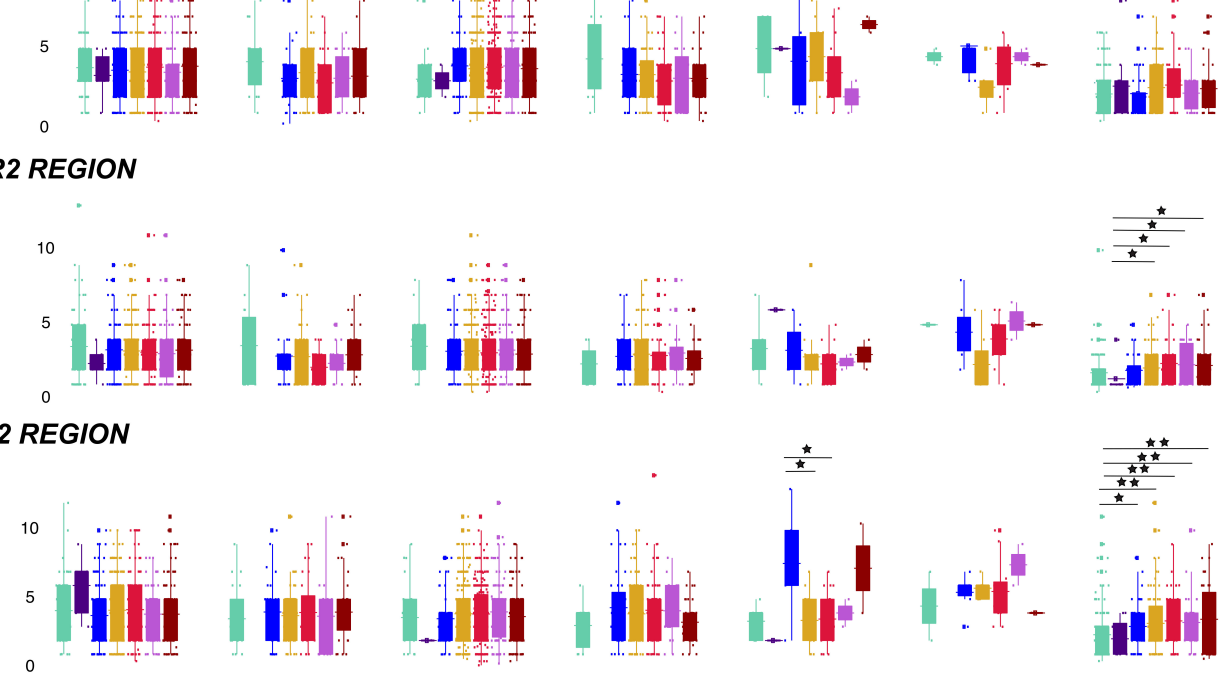

B
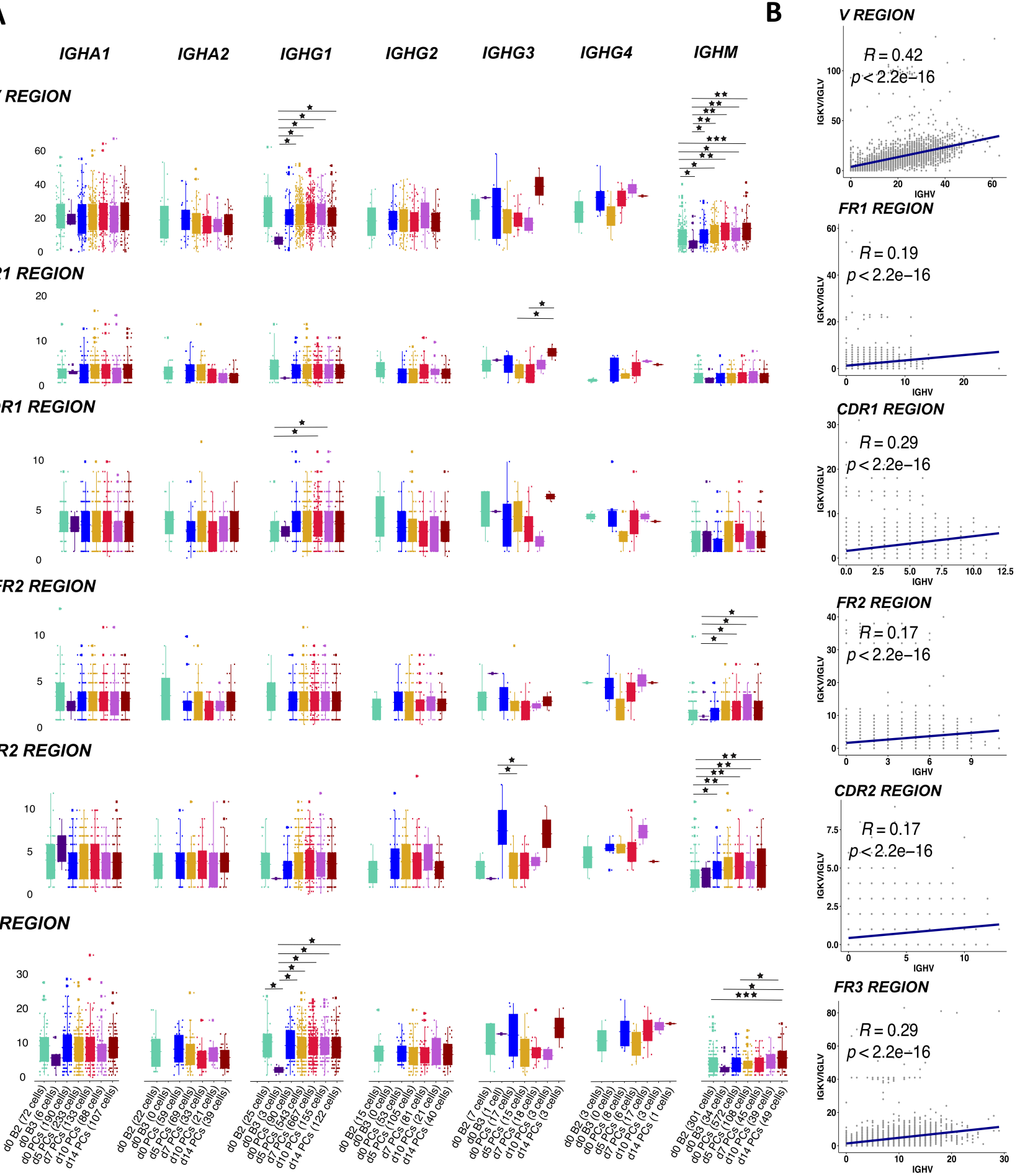

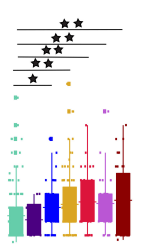

FR3 REGION

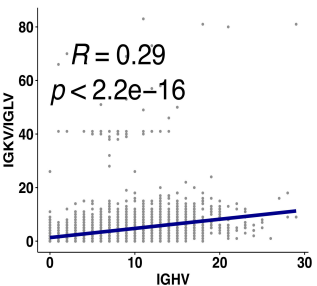

Figure 6. SHM levels in the $V$ genes in heavy and light chains. (A) The boxplot representing the number of mutations in the $V$ genes of BCRs over different time-points pre- and post-vaccination per different constant gene. The mutations have been identified in each cell over framework and complementarity determining regions in the $V$ region. The cells at $\mathrm{d} 0$ are divided over naive B cells, memory B cells, activated memory B cells and PB/PCs. Each dot is a sequence from a single cell. The significant mean differences as calculated by the $\mathrm{t}$-test are highlighted by asterix as *: 0.05-0.01, **: 0.01-0.001 and *** $<0.001$. (B) The correlation of mutation numbers in all the $V$ regions between heavy and light variable genes for all cells with known paired constant BCRs. The regression line is fitted and the correlation and $\mathrm{p}$ values are indicated on top. 


\subsection{BCRs Specific to Vaccine Toxoids}

To identify toxoid-specific lineages in the scBCR-rep, we searched literature for the CDR3 sequences known to be specific to antigens present in the Boostrix vaccine. We used the CDR3 amino acid sequences of the (mono-)clonal Abs for the anti-DT [23], antiTT [24] and anti-PT [25] to query the related BCRs in our data (Figure S6). To account for individual-specific variability in the CDR3 sequences (as a result of SHM and affinity maturation processes specific to the individuals), we allowed a certain level of flexibility in our search. Apart from relaxing the identity of the CDR3 sequence at the amino acid level to $60-70 \%$, the length of the CDR3 sequences was also allowed to vary by 1-3 amino acid residues.

\subsubsection{Identification of Conserved Anti-TT BCRs}

We could not identify any relevant BCR sequence highly related to the anti-DT Ab sequences previously published. However, we identified 54 BCRs from 25 clonotypes that were highly similar to anti-TT Ab query sequences. $65 \%$ of the observed anti-TT BCR clones used the IGHG1 constant gene followed by the IGHA1 (13\%), IGHM (15\%) and IGHA2 (6\%) genes. IGHG1 was observed at all time-points, whereas IGHA1, IGHA2 and IGHM were observed only until d7 post-vaccination. Clone 288 and 291 are $82 \%$ and $88 \%$ identical to the query anti-TT Ab sequence (Figure 7A). Similarly, clone 681 and 2083 are $80 \%$ and $86 \%$ identical to their respective anti-TT Ab query sequences. Clone 288 was the largest expanded clone with anti-TT specific BCRs (Figure 7B). This clone was present mostly at the later time-points post-vaccination. The presence of highly conserved BCR clones to the queried anti-TT Ab sequences suggests the BCRs can be highly conserved among different individuals. Furthermore, it also suggests that the immune response against TT is memorized efficiently by the immune system for secondary responses.

A

\begin{tabular}{|c|c|c|}
\hline \multicolumn{3}{|c|}{ IGHV1-18, IGHD3-10, IGHJ4 } \\
\hline TT1 & CARDYFHSGSQYFFDYW & $\mathrm{TT} 2$ \\
\hline C288_1 & CARDYFDSGSYYIFD WW & C681_1 \\
\hline C288_2 & CARDYFDSGSYYIFDYW & C681-2 \\
\hline C288_3 & CARDYFDSGSYYIFDXW & C681-3 \\
\hline C288_4 & CARDYFDSGSYYIFDYW & \\
\hline $\mathrm{C} 288{ }^{-} 5$ & CARDYFDSGSYYIFDYW & \\
\hline $\mathrm{C} 288 \_6$ & CARDYFDSGSYYIEDYW & TT3 \\
\hline $\mathrm{C} 288_{-} 7$ & CARDYFDSGSYYIFDYW & \\
\hline $\mathrm{C} 288_{-}^{-} 8$ & CARDYFDSGSYYIFDYW & 208 \\
\hline $\mathrm{C} 288_{-}^{-} 9$ & CARDYFDSGSYYIFD WW & \\
\hline C288_10 & CARDYFDSGSYYIFDYW & \\
\hline C288_11 & CARDYFDSGSYYIFDWW & \\
\hline C288_12 & CARDYFDSGSYYIFDYW & \\
\hline C288_13 & CARDYFDSGSYYIFDYW & \\
\hline C288_14 & CARDYFDSGSYYIFDXW & \\
\hline C288_15 & CARDYFDSGSYYIFDYW & \\
\hline C288_16 & CARDYFDSGSYYIFDYW & \\
\hline $291 \_1$ & CARDYFGSGSRYFFDWW & \\
\hline $291-2$ & CARDYFGSGSRYFFDWW & \\
\hline
\end{tabular}

C

PT1 CARDRRKVRGV-WAGYSG-MDVW

C2450_1 CAADRAKVRGVIIEGRVGAMDVW

C2450 2 CANDRAMVRGVVIEGRVGAMDVW

C2450 3 CVHDRAKVRGVVIEGRVGAMDVWT

C2450_4 CVADRAKVRGVVIEGRVGAMDVW
B

IGHV2-5 IGHJ4

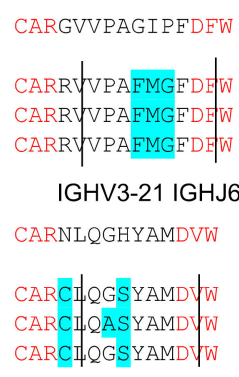

CARCHQGSYAMDYW

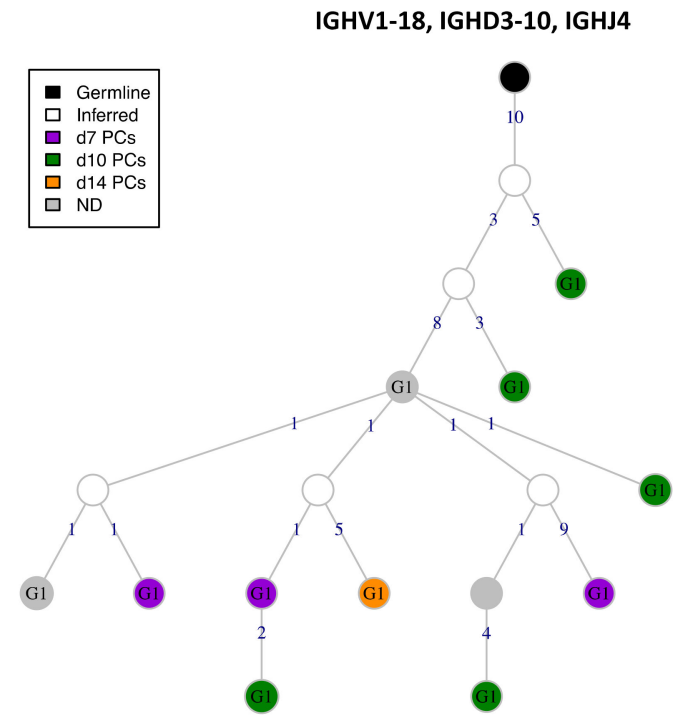

Figure 7. Toxoid-specific lineages upon Boostrix vaccination. (A) Highly conserved anti-tetanus toxoid BCR clonotypes. Four clonotypes with $>80 \%$ identity are aligned to the anti-TT Ab query sequences. The changed residues are highlighted 
in cyan and clonotype IDs are mentioned. The $V$ and $J$ gene residues are highlighted in red and demarcated by straight lines. (B) BCR lineage of anti-tetanus toxoid clonotype 288. The largest anti-TT BCR clonotype is visualized. (C) (Un-)Likely related anti-pertussis toxoid BCR clonotypes. One clonotype with $>70 \%$ identity is aligned to the respective anti-PT $\mathrm{Ab}$ query sequences. The length flexibility allowed the identification of these clonotypes. The changed residues are highlighted in cyan and the insertions are highlighted in yellow. Clonotype IDs are mentioned.

\subsubsection{Identification of BCRs (Un-)Likely Related to Anti-PT BCRs}

Analogously, we identified 20 BCRs that were similar to the anti-PT Ab query sequences, however the BCRs were not conserved as compared to the query sequences. This might suggest that the selected BCRs may be specific to antigens other than PT. 55\% of the hits used the IGHG1 gene sampled from all visits, followed by 30\% IGHM BCRs sampled from the memory B cells (cluster B3) at baseline. Unlike the anti-TT BCR sequences, we also observed a few BCRs using the IGHD constant gene. One clone with maximum identity $(\sim 70 \%)$ to the anti-PT Ab query sequence was found (Figure $7 \mathrm{C})$. This clone used the IGHG1 constant gene. Clone 2450 is $73 \%$ identical to its respective anti-PT Ab query sequence. We did not observe highly conserved (>80\%) CDR3 sequences to the anti-PT $\mathrm{Ab}$ query sequences suggesting either a unique individual-specific repertoire or recurrent adaptability of the immune response to the subsequent encounter of the antigen i.e., PT.

\section{Discussion}

Understanding human B-cell immune responses to infections is critical for vaccine development and assessment [26]. The repertoire of antigen receptor sequences changes during an encounter with foreign antigen from pathogens/vaccination [19,27]. Identifying the signatures of effective responses from the repertoire data is challenging and systematic analysis of the repertoire together with the dynamics of clonal lineages during infection/vaccination is crucial. Here, we systematically characterized the cell types in sorted B cells and PB/PCs using single-cell transcriptomics data and developed a robust workflow to capture the longitudinal changes (d0, 5, 7, 10, and 14 after Boostrix vaccination) in the BCRs and clonal lineages using repertoire data from the same cells. Moreover, we accorded our results with previously acquired flow cytometry data from the same individual [5]. We identified additional membrane markers that could be used in flow cytometry to dissect the maturation of PB/PCs. Furthermore, using one individual, we developed a bioinformatic workflow as a proof-of-principle to compare the BCR-rep in PB/PCs with the baseline B-cell repertoire and reported expansion of unique clones in post-vaccination visits, high usage of IGHG1 in expanded clones, increased class switching events post-vaccination and positive selection of CDR3 sequences over time. We also found that the Variable gene family-based clustering of BCRs improved the clustering of BCRs. Finally, the query tool developed in the study dissect the immune response to the components of Boostrix vaccine is unique to this study. Albeit that this pilot study contains only one individual, this study still allows us to select for the right processes to be evaluated in a larger series. Additionally, the analysis performed in one individual could impact the biological findings in our study, especially, the $V(D) J$ gene usage and clonal lineage analysis, which should be assessed in larger studies.

The classical markers used in phenotyping by flow cytometry are not always captured in the sparse single-cell transcriptomics data which makes it challenging to define uniform populations in both the technologies. Therefore, the identification of naive B cells, memory B cells, activated memory B cells and plasma cell clusters was made using a combination of classical CD markers and other gene-based markers known in the literature. For example, CXCR4, a chemokine receptor, turned out to be an important marker for the identification of the naive B cells [16], whereas the classical markers IGHD, CD19 were dimly expressed in this cell subset. Also, CXCR4 is known to be progressively downregulated in transition from naive B cells to memory B cells to activated memory B cells [19], which we also observed in clusters B1, B2 and B3 (Figure 1D). The activated memory B-cell state displays a hallmark of an effector B-cell response and shares several markers with previously 
defined aged/autoimmunity-associated B cells, e.g., high expression of ITGAX (CD11c) [28]. Another activated memory B-cell marker, i.e., ZBTB32, is known to modulate the duration of memory B-cell recall responses in mice [29]. Defining the B-cell populations accurately helps in better understanding of the molecular processes e.g., immunoglobulin production, antigen processing and presentation and glycolysation in vaccination/infection (Figure 2F), which in turn might be helpful in identifying therapeutic targets in pathogen immunity. Moreover, among all activated memory B-cell markers, ITGAX, CD72 and TNFRSF1B are membrane markers, that in addition to the classical B-cell markers can be used to sort activated memory B cells.

Despite regressing out cell cycle genes, we still observed a high score for the $\mathrm{G} 2+\mathrm{S}$ genes in the P4 cluster, suggestive of the most proliferating PB/PCs. Although we did not consider the cell cycle genes for lineage tracing, the P4 plasma cell cluster was the endpoint of the trajectory, which suggests the role of developmental genes other than cell cycle genes in the plasma cell development and proliferation. Interestingly, these P1-P4 clusters of $\mathrm{PB} / \mathrm{PC}$ do not accurately depict the developmental stages of PB/PCs as defined by the $C D 20$ and CD138 markers in flow cytometry [5]. We showed that using flow cytometry data as a reference, the stages of plasma cell maturation could be tracked accurately and additional plasma cell maturation markers, contributing from the early to late plasma cell maturation pathway, were identified. For example, peroxiredoxin PRDX3 (similar expression as SELL (CD62L)) suffices the metabolic requirements of PCs in early phase of development that are destined to secrete massive amounts of Ig [30]. PPIA, peptidyl-prolyl isomerase $\mathrm{A}$, catalyzes the protein folding and might play an important role in folding the Abs in the early phase of plasma cell development. Additionally, PABPC1 (expression similar to $C D 19)$ mediates the regulation of the switching from membrane $\operatorname{IgH}$ to secreted IgH isoform in PCs [31]. CD74 (expression similar to MS4A1 (CD20)) is directly involved in shaping the scBCR-rep by initiating a cascade that results in proliferation of $B$ cells and rescues the cells from apoptosis [32,33]. MZB1 (expression similar to CD27) is an important co-chaperone in plasma cell differentiation [34]. Loss of this gene can affect migratory properties of the Ab secreting cells and their trafficking and retention to the bone marrow [34]. Similarly, the SSR4 gene plays an important role in protein assembly and trafficking [35]. Its high expression is related to IgG4-related diseases [36]. The markers DDOST and PPIB (expression similar to CD38) are involved in protein glycosylation and unfolded protein responses in the plasma cell development process as also identified in the pathway analysis. Finally, XBP1 (similar expression as SDC1 (CD138)) is responsible for late events i.e., increased protein synthesis in plasma cell differentiation $[37,38]$. Herein, $32 \%$ of 104 genes were found to express membrane bound proteins and hence can be used in flow cytometry to dissect additional stages of plasma cell maturation and/or sort specific populations e.g., Ab-secreting cells. A few of these markers e.g., CD74 [39] and CD22 [40] have already been used in quantitative flow cytometry studies to distinguish normal $\mathrm{B}$ cells from the B-cell related malignancies.

The repertoire in response to infection/vaccination has generally preference for specific $V, D$ or $J$ genes. For example, anti-hemagglutinin clones have been shown to use the IGHV1-69 gene [35,41] and IGHV4-34 gene [19] is abundant in influenza vaccination/infection studies. However, the usage of the IGHV3-7 gene increased after Hepatitis B vaccination [42]. Also, the abundance of the IGHV4-59, IGHV4-39, IGHV3-23, IGHV3-53, IGH3-66, IGHV2-5, and IGHV2-70 genes was found in SARS-CoV2 infection studies [43-46]. Similarly, we observed an increased usage of the IGHV3-23 gene on all visits post-vaccination; the usage of the IGHV1-2, IGHV1-18, IGHV3-33, IGHV4-39, IGHV4-31 genes increased at d5, d7 and d10 post-vaccination; and IGHV5-51 was highly used at d10 post-vaccination. We found that none of the specific classes of the IGHV genes has driven the overall response. Moreover, we identified high usage of the IGHD3 family and the IGHJ6 gene, which is in general abundantly used in the general repertoire [47-49], most likely owing to the perfect recombination signal sequences [50,51]. In the light chains, we also observed a high usage of the IGK genes as compared to the IGL loci gene, reflecting 
the normal 3:2 ratio observed in humans at baseline [52-54], which was skewed to a $\sim 8: 3$ ratio at $\mathrm{d} 5$, suggesting selection mechanisms due to the antigen exposure.

We identified $\sim 900$ of $\sim 3500$ clonotypes with $>1$ BCR clustered together. The majority of them were present on $\mathrm{d} 5$ and $\mathrm{d} 7$ post-vaccination. Although, the PB/PCs expanded after vaccination are enriched for vaccine-specificity, we cannot rule out that some of the PCs may have a different specificity $[55,56]$. Approximately, $70 \%$ of the Ab-secreting cells in the influenza vaccination study have been shown to be influenza-vaccine specific, previously [8]. The clonotype classification allowed us to identify class-switching events, the majority of which contained IGHM and IGHG, followed by IGHM and IGHA. It is known from previous studies that $\sim 85 \%$ of the switches from IgM commonly comprises of IgG1, IgA1 and IgG2 in decreasing order [57]. Higher usage of the IgG subclasses (48\% IgG1, 22\% IgG2, and 9\% IgG3) has been known previously from influenza vaccine responsive clones [19], which was also observed in our study. Altogether, the highest usage of the IGHG1 gene and the highest number of clonotypes containing IgM and IgG suggest the vaccine target IgG-based immune response, common immune response in intramuscular vaccine administration.

The selection processes and molecular events affect the $V$ gene of $I G$ heavy chain differently than the light chains $[58,59]$ resulting in less diversity in the light chain repertoire [60]. Although we observed significant correlations between the mutations in $V$ regions in heavy and light chain genes, the correlation was $<45 \%$. The high mutation rate in IGHV BCRs utilizing the IGHM class has been proposed as a mechanism of the immune system to maintain long-term memory to the vaccine [61]. Moreover, the high number of mutations in IGHG1 and IGHM genes in CDR and FR3 regions might also be associated with the maturation of IgG affinity during the response to the Boostrix vaccine. As the vaccine is a booster and only one individual was used for the pilot study, it might be difficult to assess the role of booster in affinity maturation processes.

The dissection of the immune response to all the antigens and/or separate components of a vaccine, when cells are not pre-sorted based on their reactivity, can be helpful to estimate the response to each of the antigens. We identified a few clonotypes related to anti-TT and anti-PT BCRs, suggesting the public nature of the queried sequences. Identification of highly conserved anti-TT BCRs suggests an optimal repertoire for strong immunodominant epitopes. However, we observed that the anti-PT BCRs were not highly conserved which could be explained by either a unique private repertoire to the antigen or that each encounter with this antigen shapes the repertoire with additional mutations. The number of identified anti-TT and anti-PT clones are not directly related to the complexity of the proteins in the vaccine but related to the number of epitopes found in the literature. Additional support to this observation can be obtained with repertoire data obtained from antigen-specific $B$ cells from multiple individuals.

Overall, we identified B-cell populations and additional plasma cell maturation pathway biomarkers based on the transcriptomics data. Importantly, we used the longitudinal scBCR-rep data to understand the $V(D) J C$ gene usage, clonal expansion and SHM events post-vaccination and their role in shaping immune responses. Together, we were able to dissect the immune responses to the components of the Boostrix vaccine.

\section{Conclusions}

To conclude, we have characterized the PB/PCs response of an immunized donor by performing longitudinal analysis ( $\mathrm{d} 0,5,7,10$, and 14 after Boostrix vaccination) of the single-cell transcriptome as well as the B-cell receptor (BCR) repertoire (scBCR-rep) data and comparing them with the flow cytometry data from the same donor. We report that the conventional membrane protein markers for cell type identification in flow cytometry do not match transcript expression levels. Hence, proper B-cell subsetting based on gene expression profile requires use of additional parameters. Also, the flowcytometry data guided us to trace the maturation pathway of the $\mathrm{PB} / \mathrm{PCs}$, which was not feasible with the single-cell transcriptomic data, owing to differences in the transcript level expression. 
However, after tracing the maturation pathway, we could find multiple additional membrane markers e.g., CD37, CD52, CD22, CD53, FCER2 that could be used in flow cytometry to dissect the maturation of PB/PCs. Furthermore, in this study, we developed a methodology to compare the BCR-rep in PB/PCs with the baseline B-cell repertoire and reported expansion of unique clones in post-vaccination visits, high usage of IGHG1 in expanded clones, increased class-switching events post-vaccination and positive selection of CDR3 sequences over time. We also found that the Variable gene family-based clustering of BCRs improved the clustering of BCRs. Finally, the query tool developed in the study to dissect the immune response to the components of the Boostrix vaccine is unique to this study.

Supplementary Materials: The following are available online at https://www.mdpi.com/article/10 .3390 / vaccines9111352/s1, Figure S1: Overview of the time-points and the single-cell sorting strategy of samples obtained pre and post-vaccination from a single individual. (A) One healthy female volunteer (age 28; primed with DTP in infancy with no history of pertussis infection or vaccination in the past 10 years) was vaccinated using Boostrix vaccine. Peripheral blood samples were collected at d0 (baseline), d5, d7, d10 and d14 after vaccination. (B) Workflow to sort 10,000 baseline B cells from baseline, and all PB/PCs from baseline, d5, d7, d10 and d14. The collection Eppendorf tube was precoated overnight with a 5\% BSA solution (airdried afterwards) to reduce adherence of cells to the plastic. B cells were defined as Zombie-CD19+ cells. PB/PCs were defined as ZombieCD19+CD38++CD24+CD27+. Created on Biorender.com; accessed on 4 October 2021. (C) Total B and $\mathrm{PB} / \mathrm{PC}$ numbers of this donor as determined by flow cytometry. The dashed lines indicate the baseline reference values for age-matched individuals (as described by Blanco et al., 2018 [62]). $\mathrm{Ab}$; antibody, $\mathrm{mAb}$; monoclonal $\mathrm{Ab}, \mathrm{L} / \mathrm{D}$; life/dead marker, d; days after Boostrix vaccination. Figure S2: Identification of singlets in the CITE-Seq single-cell data based on the expression of HTOs. The low-quality cells with unique features $<100$ or $>5000$ features, or/and $>25 \%$ mitochondrial content were filtered out. After quality filtering steps, MULTIseqDemux was used to identify the singlets and match the singlets to respective tags hence visits. A tSNE of the singlets based on the tag expression is shown to depict the separation between tags. Figure S3: The number of recorded IG gene rearrangements and the $V(D) J$ usage across different visits. (A) The tSNE plot of the cells colored based on the number of recorded IG gene rearrangements. The bar graph is used to indicate the numbers of heavy and light chains retained after filtering per cell. (B) The usage of heavy and light variable genes across each visit. The numbers in the heatmap are the number of chains each combination of genes and the colors are ranged based on the scaled counts for each visit. (C) The alluvial plots to depict the usage of $V(D) J$ genes in heavy chains. The counts of each $V(D) J$ usage are scaled (min:0, max:1) for comparison. The width of strata represents higher usage of the gene family. (D) Circos plot of the $V$ and $J$ genes in the heavy chains at d0 B cells and PB/PCs. Figure S4: BCR clonotype expansions over different time-points. (A) Top 30 most numerous clonotypes (909 BCRs) identified from 6347 cells over different time-points pre- and post-vaccination. The count of clones per visit is colored. N.D. represent the receptors with no recorded visits. (B) BCR clonal lineage of clonotype 660. The top third clonotype 660 with more than 50 BCRs is represented. Each BCR in the clonotype uses IGHG1 and is different from its neighbors by one to 18 mutations, represented as edge labels. (C) CDR3 length distribution of the BCR amino acid sequences over different time-points. N.D. represent the receptors with no recorded visits. Figure S5: Quantification of antigen-driven selection over-time post-vaccination in PB/PCs. The top panel depicts the density plot of the probability distribution of selection in CDR and FWR region in PB/PCs at baseline and all time-points post-vaccination. Negative sigma value indicates negative selection, neutral at sigma zero while positive value indicates positive selection. The plot in lower panel is simplified view of sigma values plotted for each time-points in both CDR and FWR regions. In the lower plot, we can clearly observe a neutral selection in FWR region in d10 PCs. Figure S6: The workflow of the query tool to identify toxoid-specific lineages in the scBCR-rep data. The input sequences are searched against the database of consensus sequences of each clonotype. A LD score was obtained for each query sequence against similar consensus sequences (same $V$ family, same $J$ gene; with user-defined CDR3 length adjusted and identity). The LD score is used to finally compile the related consensus sequence and the clonotypes for each query sequence. Table S1: List of genes with similar expression to the 6 maturation genes. The gene names highlighted in green corresponds to the membrane markers. The dark grey shading indicates no pathway was associated with the gene, whereas light 
grey shading indicates the absent pathways. Table S2: Number of clusters identified by different grouping criteria with $V$ genes and family. The table is divided into three blocks based on varying BCR counts in clusters based on gene or family definition. The first block shows the number of clusters that actually differed between gene and family definitions; the second block represent unique clusters, whereas the last block, highlighted in yellow, represent the clusters that stayed constant even if the definition changed. The adjusted rand index for both the clustering methods was 0.99 suggesting very similar clustering output between the two definitions.

Author Contributions: I.K., A.M.D., E.B.v.d.A., M.J.T.R., J.J.M.v.D. and M.A.B.: concept and design of the study. A.M.D. and M.A.B.: Processed samples for single-cell sequencing and organized clinical part of the study. I.K.: Analyzed the data. I.K. and M.A.B.: Interpreted the results. L.E.M.O. and J.J.Z. coordinated the clinical part of this study. All authors have read and agreed to the published version of the manuscript.

Funding: This project has received funding from the PERISCOPE program. PERISCOPE has received funding from the Innovative Medicines Initiative 2 Joint Undertaking under grant agreement No 115910. This Joint Undertaking receives support from the European Union's Horizon 2020 research and innovation program and European Federation of Pharmaceutical Industries and Associations (EFPIA) and Bill and Melinda Gates Foundation (BMGF). IK received personal funding for this work from the European Union's Horizon 2020 research and innovation program under the Marie Skłodowska-Curie grant agreement No 707404.

Institutional Review Board Statement: The study was conducted according to the guidelines of the Declaration of Helsinki, and approved by the Medical Ethics Committee Leiden-Den Haag-Delft (protocol code P16-214, EUDRACT:2016-002011-18, approved on 11-01-2017).

Informed Consent Statement: Informed consent was obtained from all subjects involved in the study.

Data Availability Statement: Raw and processed data have been deposited at Gene Expression Omnibus and are available via GEO accession GSE185427. The codes are made available via the GitHub repository https://github.com/InduKhatri/Single-cell-BCR.

Acknowledgments: The authors gratefully acknowledge the Flow cytometry Core Facility at LUMC (coordinated by K. Schepers, M. Hameetman, run by operators S. van de Pas, D. Lowie, J. Jansen, IJ. Reyneveld and former operators E. de Haas and G. de Roo) for their support. We are grateful to the subject, who volunteered to contribute to clinical study. We would also like to acknowledge Rick J. Groenland and Bas de Mooij, who helped in acquiring and sorting cells for the study. We would like to acknowledge Susan Kloet and the personnel from the Leiden genome technology center (LGTC) at the LUMC for providing the sequencing support. Finally, the authors would like to thank the team of Liesbeth Oosten and Jaap-Jan Zwaginga from the Dept. of Hematology, LUMC, for their help with the clinical part of this study.

Conflicts of Interest: J. J. M. van Dongen is the founder of the EuroClonality Consortium and one of the inventors on the EuroClonality-owned patents and EuroFlow-owned patents, which are licensed to Invivoscribe, BD Biosciences or Cytognos; these companies pay royalties to the EuroClonality and EuroFlow Consortia, respectively, which are exclusively used for sustainability of these consortia. J. J. M. van Dongen reports an Educational Services Agreement with BD Biosciences and a Scientific Advisory Agreement with Cytognos, with honorarium fees paid to LUMC. The rest of the authors declare that they have no other relevant conflicts of interest.

\section{References}

1. Eisen, H.N. Affinity enhancement of antibodies: How low-affinity antibodies produced early in immune responses are followed by high-affinity antibodies later and in memory B-cell responses. Cancer Immunol. Res. 2014, 2, 381-392. [CrossRef] [PubMed]

2. Hammarlund, E.; Thomas, A.; Amanna, I.J.; Holden, L.A.; Slayden, O.D.; Park, B.; Gao, L.; Slifka, M.K. Plasma cell survival in the absence of B cell memory. Nat. Commun. 2017, 8, 1781. [CrossRef]

3. Imani, F.; Kehoe, K.E. Infection of human B lymphocytes with MMR vaccine induces IgE class switching. Clin. Immunol. 2001, 100, 355-361. [CrossRef]

4. Pan-Hammarström, Q.; Zhao, Y.; Hammarström, L. Class Switch Recombination: A Comparison Between Mouse and Human. Adv. Immunol. 2007, 93, 1-61.

5. Diks, A.M.; Khatri, I.; Oosten, L.E.; De Mooij, B.; Groenland, R.J.; Teodosio, C.; Perez-Andres, M.; Orfao, A.; Berbers, G.A.M.; Zwaginga, J.J.; et al. Highly sensitive flow cytometry allows monitoring of changes in circulating immune cells in blood after Tdap booster vaccination. Front. Immunol. 2021, 12, 2091. [CrossRef] 
6. GlaxoSmithKline. BOOSTRIX (Tetanus Toxoid, Reduced Diphtheria Toxoid and Acellular Pertussis Vaccine, Adsorbed) Injectable Suspension, for Intramuscular Use; GlaxoSmithKline: Research Triangle Park, NC, USA, 2020.

7. Ellebedy, A.H.; Jackson, K.J.L.; Kissick, H.T.; Nakaya, H.I.; Davis, C.W.; Roskin, K.M.; McElroy, A.K.; Oshansky, C.M.; Elbein, R.; Thomas, S.; et al. Defining antigen-specific plasmablast and memory B cell subsets in human blood after viral infection or vaccination. Nat. Immunol. 2016, 17, 1226-1234. [CrossRef]

8. Wrammert, J.; Smith, K.; Miller, J.; Langley, W.A.; Kokko, K.; Larsen, C.; Zheng, N.Y.; Mays, I.; Garman, L.; Helms, C.; et al. Rapid cloning of high-affinity human monoclonal antibodies against influenza virus. Nature 2008, 453, 667-671. [CrossRef]

9. Stuart, T.; Butler, A.; Hoffman, P.; Hafemeister, C.; Papalexi, E.; Mauck, W.M.; Hao, Y.; Stoeckius, M.; Smibert, P.; Satija, R. Comprehensive Integration of Single-Cell Data. Cell 2019, 177, 1888-1902.e21. [CrossRef] [PubMed]

10. Mylka, V.; Aerts, J.; Matetovici, I.; Poovathingal, S.; Vandamme, N.; Seurinck, R.; Hulselmans, G.; Van Den Hoecke, S.; Wils, H.; Reumers, J.; et al. Comparative analysis of antibody-and lipid-based multiplexing methods for single-cell RNA-seq. bioRxiv 2020. [CrossRef]

11. Kernfeld, E.M.; Genga, R.M.J.; Neherin, K.; Magaletta, M.E.; Xu, P.; Maehr, R. A Single-Cell Transcriptomic Atlas of Thymus Organogenesis Resolves Cell Types and Developmental Maturation. Immunity 2018, 48, 1258-1270.e6. [CrossRef] [PubMed]

12. Bindea, G.; Mlecnik, B.; Hackl, H.; Charoentong, P.; Tosolini, M.; Kirilovsky, A.; Fridman, W.H.; Pagès, F.; Trajanoski, Z.; Galon, J. ClueGO: A Cytoscape plug-in to decipher functionally grouped gene ontology and pathway annotation networks. Bioinformatics 2009, 25, 1091-1093. [CrossRef]

13. Bischof, J.; Ibrahim, S.M. bcRep: R Package for Comprehensive Analysis of B Cell Receptor Repertoire Data. PLoS ONE 2016, 11, e0161569. [CrossRef]

14. Zuckerman, N.S.; Hazanov, H.; Barak, M.; Edelman, H.; Hess, S.; Shcolnik, H.; Dunn-Walters, D.; Mehr, R. Somatic hypermutation and antigen-driven selection of B cells are altered in autoimmune diseases. J. Autoimmun. 2010, 35, 325-335. [CrossRef]

15. Stern, J.N.H.; Yaari, G.; Vander Heiden, J.A.; Church, G.; Donahue, W.F.; Hintzen, R.Q.; Huttner, A.J.; Laman, J.D.; Nagra, R.M.; Nylander, A.; et al. B cells populating the multiple sclerosis brain mature in the draining cervical lymph nodes. Sci. Transl. Med. 2014, 6, 248ra107. [CrossRef]

16. Berkowska, M.A.; Schickel, J.-N.; Grosserichter-Wagener, C.; de Ridder, D.; Ng, Y.S.; van Dongen, J.J.M.; Meffre, E.; van Zelm, M.C. Circulating Human CD27 - IgA + Memory B Cells Recognize Bacteria with Polyreactive Igs. J. Immunol. 2015, 195, 1417-1426. [CrossRef] [PubMed]

17. Toellner, K.M. FOXP1 inhibits plasma cell differentiation. Blood 2015, 126, 2076-2077. [CrossRef]

18. Hövelmeyer, N.; Wörns, M.A.; Reissig, S.; Adams-Quack, P.; Leclaire, J.; Hahn, M.; Wörtge, S.; Nikolaev, A.; Galle, P.R.; Waisman, A. Overexpression of Bcl-3 inhibits the development of marginal zone B cells. Eur. J. Immunol. 2014, 44, 545-552. [CrossRef] [PubMed]

19. Horns, F.; Vollmers, C.; Dekker, C.L.; Quake, S.R. Signatures of selection in the human antibody repertoire: Selective sweeps, competing subclones, and neutral drift. Proc. Natl. Acad. Sci. USA 2019, 116, 1261-1266. [CrossRef]

20. Shi, Z.; Zhang, Q.; Yan, H.; Yang, Y.; Wang, P.; Zhang, Y.; Deng, Z.; Yu, M.; Zhou, W.; Wang, Q.; et al. More than one antibody of individual B cells revealed by single-cell immune profiling. Cell Discov. 2019, 5, 64. [CrossRef]

21. Yaari, G.; Uduman, M.; Kleinstein, S.H. Quantifying selection in high-throughput Immunoglobulin sequencing data sets. Nucleic Acids Res. 2012, 40, e134. [CrossRef] [PubMed]

22. Fang, L.; Lowther, D.E.; Meizlish, M.L.; Anderson, R.C.E.; Bruce, J.N.; Devine, L.; Huttner, A.J.; Kleinstein, S.H.; Lee, J.Y.; Stern, J.N.H.; et al. The immune cell infiltrate populating meningiomas is composed of mature, antigen-experienced $\mathrm{T}$ and $\mathrm{B}$ cells. Neuro. Oncol. 2013, 15, 1479-1490. [CrossRef]

23. Sevigny, L.M.; Booth, B.J.; Rowley, K.J.; Leav, B.A.; Cheslock, P.S.; Garrity, K.A.; Sloan, S.E.; Thomas, W.; Babcock, G.J.; Wang, Y. Identification of a human monoclonal antibody to replace equine diphtheria antitoxin for treatment of diphtheria intoxication. Infect. Immun. 2013, 81, 3992-4000. [CrossRef]

24. Lavinder, J.J.; Wine, Y.; Giesecke, C.; Ippolito, G.C.; Horton, A.P.; Lungu, O.I.; Hoi, K.H.; DeKosky, B.J.; Murrin, E.M.; Wirth, M.M.; et al. Identification and characterization of the constituent human serum antibodies elicited by vaccination. Proc. Natl. Acad. Sci. USA 2014, 111, 2259-2264. [CrossRef]

25. Acquaye-Seedah, E.; Reczek, E.E.; Russell, H.H.; DiVenere, A.M.; Sandman, S.O.; Collins, J.H.; Stein, C.A.; Whitehead, T.A.; Maynard, J.A. Characterization of individual human antibodies that bind pertussis toxin stimulated by acellular immunization. Infect. Immun. 2018, 86, e00004-18. [CrossRef]

26. Wec, A.Z.; Haslwanter, D.; Abdiche, Y.N.; Shehata, L.; Pedreño-Lopez, N.; Moyer, C.L.; Bornholdt, Z.A.; Lilov, A.; Nett, J.H.; Jangra, R.K.; et al. Longitudinal dynamics of the human B cell response to the yellow fever 17D vaccine. Proc. Natl. Acad. Sci. USA 2020, 117, 6675-6685. [CrossRef]

27. Nourmohammad, A.; Otwinowski, J.; Łuksza, M.; Mora, T.; Walczak, A.M.; Leitner, T. Fierce Selection and Interference in B-Cell Repertoire Response to Chronic HIV-1. Mol. Biol. Evol. 2019, 36, 2184-2194. [CrossRef] [PubMed]

28. Hao, Y.; O'neill, P.; Naradikian, M.S.; Scholz, J.L.; Cancro, M.P. A B-cell subset uniquely responsive to innate stimuli accumulates in aged mice. Blood J. Am. Soc. Hematol. 2011, 118, 1294-1304. [CrossRef] [PubMed]

29. Jash, A.; Wang, Y.; Weisel, F.J.; Scharer, C.D.; Boss, J.M.; Shlomchik, M.J.; Bhattacharya, D. ZBTB32 Restricts the Duration of Memory B Cell Recall Responses. J. Immunol. 2016, 197, 1159-1168. [CrossRef] [PubMed] 
30. Masciarelli, S.; Sitia, R. Building and operating an antibody factory: Redox control during B to plasma cell terminal differentiation. Biochim. Biophys. Acta-Mol. Cell Res. 2008, 1783, 578-588. [CrossRef]

31. Peng, Y.; Yuan, J.; Zhang, Z.; Chang, X. Cytoplasmic poly(A)-binding protein 1 (PABPC1) interacts with the RNA-binding protein hnRNPLL and thereby regulates immunoglobulin secretion in plasma cells. J. Biol. Chem. 2017, 292, 12285-12295. [CrossRef] [PubMed]

32. Cohen, S.; Shachar, I. Cytokines as regulators of proliferation and survival of healthy and malignant peripheral B cells. Cytokine 2012, 60, 13-22. [CrossRef]

33. Gil-Yarom, N.; Radomir, L.; Sever, L.; Kramer, M.P.; Lewinsky, H.; Bornstein, C.; Blecher-Gonen, R.; Barnett-Itzhaki, Z.; Mirkin, V.; Friedlander, G.; et al. CD74 is a novel transcription regulator. Proc. Natl. Acad. Sci. USA 2017, 114, 562-567. [CrossRef]

34. Andreani, V.; Ramamoorthy, S.; Pandey, A.; Lupar, E.; Nutt, S.L.; Lämmermann, T.; Grosschedl, R. Cochaperone Mzb1 is a key effector of Blimp1 in plasma cell differentiation and $\beta 1$-integrin function. Proc. Natl. Acad. Sci. USA 2018, 115, E9630-E9639. [CrossRef]

35. Lingwood, D.; McTamney, P.M.; Yassine, H.M.; Whittle, J.R.R.; Guo, X.; Boyington, J.C.; Wei, C.J.; Nabel, G.J. Structural and genetic basis for development of broadly neutralizing influenza antibodies. Nature 2012, 489, 566-570. [CrossRef]

36. Lin, W.; Zhang, P.; Chen, H.; Chen, Y.; Yang, H.; Zheng, W.; Zhang, X.; Zhang, F.; Zhang, W.; Lipsky, P.E. Circulating plasmablasts/plasma cells: A potential biomarker for IgG4-related disease. Arthritis Res. Ther. 2017, 19, 25. [CrossRef]

37. Shaffer, A.L.; Shapiro-Shelef, M.; Iwakoshi, N.N.; Lee, A.H.; Qian, S.B.; Zhao, H.; Yu, X.; Yang, L.; Tan, B.K.; Rosenwald, A.; et al. XBP1, downstream of Blimp-1, expands the secretory apparatus and other organelles, and increases protein synthesis in plasma cell differentiation. Immunity 2004, 21, 81-93. [CrossRef]

38. Todd, D.J.; McHeyzer-Williams, L.J.; Kowal, C.; Lee, A.H.; Volpe, B.T.; Diamond, B.; McHeyzer-Williams, M.G.; Glimcher, L.H. $\mathrm{XBP1}$ governs late events in plasma cell differentiation and is not required for antigen-specific memory B cell development. J. Exp. Med. 2009, 206, 2151-2159. [CrossRef] [PubMed]

39. Bergmann, H.; Yabas, M.; Short, A.; Miosge, L.; Barthel, N.; Teh, C.E.; Roots, C.M.; Bull, K.R.; Jeelall, Y.; Horikawa, K.; et al. B cell survival, surface BCR and BAFFR expression, CD74 metabolism, and CD8- dendritic cells require the intramembrane endopeptidase SPPL2A. J. Exp. Med. 2013, 210, 31. [CrossRef]

40. Jasper, G.A.; Arun, I.; Venzon, D.; Kreitman, R.J.; Wayne, A.S.; Yuan, C.M.; Marti, G.E.; Stetler-Stevenson, M. Variables affecting the quantitation of CD22 in neoplastic B cells. Cytom. Part B Clin. Cytom. 2011, 80B, 83-90. [CrossRef] [PubMed]

41. Pappas, L.; Foglierini, M.; Piccoli, L.; Kallewaard, N.L.; Turrini, F.; Silacci, C.; Fernandez-Rodriguez, B.; Agatic, G.; GiacchettoSasselli, I.; Pellicciotta, G.; et al. Rapid development of broadly influenza neutralizing antibodies through redundant mutations Nature 2014, 516, 418-422. [CrossRef] [PubMed]

42. Galson, J.D.; Trück, J.; Clutterbuck, E.A.; Fowler, A.; Cerundolo, V.; Pollard, A.J.; Lunter, G.; Kelly, D.F. B-cell repertoire dynamics after sequential hepatitis B vaccination and evidence for cross-reactive B-cell activation. Genome Med. 2016, 8, 68. [CrossRef]

43. Montague, Z.; Lv, H.; Otwinowski, J.; Wu, N.C.; Nourmohammad, A.; Ka, C.; Mok, P.; Dewitt, W.S.; Isacchini, G.; Yip, G.K.; et al. Dynamics of $B$ cell repertoires and emergence of cross-reactive responses in patients with different severities of COVID-19. Cell Rep. 2021, 35, 109173. [CrossRef] [PubMed]

44. Pinto, D.; Park, Y.J.; Beltramello, M.; Walls, A.C.; Tortorici, M.A.; Bianchi, S.; Jaconi, S.; Culap, K.; Zatta, F.; De Marco, A.; et al. Cross-neutralization of SARS-CoV-2 by a human monoclonal SARS-CoV antibody. Nature 2020, 583, 290-295. [CrossRef] [PubMed]

45. Rogers, T.F.; Zhao, F.; Huang, D.; Beutler, N.; Burns, A.; He, W.T.; Limbo, O.; Smith, C.; Song, G.; Woehl, J.; et al. Isolation of potent SARS-CoV-2 neutralizing antibodies and protection from disease in a small animal model. Science 2020, 369, 956-963. [CrossRef]

46. Brouwer, P.J.M.; Caniels, T.G.; van der Straten, K.; Snitselaar, J.L.; Aldon, Y.; Bangaru, S.; Torres, J.L.; Okba, N.M.A.; Claireaux, M.; Kerster, G.; et al. Potent neutralizing antibodies from COVID-19 patients define multiple targets of vulnerability. Science 2020, 369, 643-650. [CrossRef] [PubMed]

47. Li, A.; Rue, M.; Zhou, J.; Wang, H.; Goldwasser, M.A.; Neuberg, D.; Dalton, V.; Zuckerman, D.; Lyons, C.; Silverman, L.B.; et al. Utilization of Ig heavy chain variable, diversity, and joining gene segments in children with B-lineage acute lymphoblastic leukemia: Implications for the mechanisms of VDJ recombination and for pathogenesis. Blood 2004, 103, 4602-4609. [CrossRef]

48. Yamada, M.; Wasserman, R.; Reichard, B.A.; Shane, S.; Caton, A.J.; Rovera, G. Preferential utilization of specific immunoglobulin heavy chain diversity and joining segments in adult human peripheral blood B lymphocytes. J. Exp. Med. 1991, 173, 395-407. [CrossRef]

49. Hong, B.; Wu, Y.; Li, W.; Wang, X.; Wen, Y.; Jiang, S.; Dimitrov, D.S.; Ying, T. In-depth analysis of human neonatal and adult IgM antibody repertoires. Front. Immunol. 2018, 9, 128. [CrossRef]

50. Khatri, I.; Berkowska, M.A.; van den Akker, E.B.; Teodosio, C.; Reinders, M.J.T.; van Dongen, J.J.M. Population matched (pm) germline allelic variants of immunoglobulin (IG) loci: Relevance in infectious diseases and vaccination studies in human populations. Genes Immun. 2021, 22, 172-186. [CrossRef]

51. Hesse, J.E.; Lieber, M.R.; Mizuuchi, K.; Gellert, M. V(D)J recombination: A functional definition of the joining signals. Genes Dev. 1989, 3, 1053-1061. [CrossRef]

52. Belessi, C.; Stamatopoulos, K.; Hadzidimitriou, A.; Hatzi, K.; Smilevska, T.; Stavroyianni, N.; Marantidou, F.; Paterakis, G.; Fassas, A.; Anagnostopoulos, A.; et al. Analysis of expressed and non-expressed IGK locus rearrangements in chronic lymphocytic leukemia. Mol. Med. 2005, 11, 52-58. [CrossRef] [PubMed] 
53. Bräuninger, A.; Goossens, T.; Rajewsky, K.; Küppers, R. Regulation of Immunoglobulin Light Chain Gene Rearrangements during Early B Cell Development in the Human. Eur. J. Immunol. 2001, 31, 3631-3637. [CrossRef]

54. Korsmeyer, S.J.; Hieter, P.A.; Ravetch, J.V.; Poplack, D.G.; Waldmann, T.A.; Leder, P. Developmental hierarchy of immunoglobulin gene rearrangements in human leukemic pre-B-cells. Proc. Natl. Acad. Sci. USA 1981, 78, 7096-7100. [CrossRef]

55. Odendahl, M.; Mei, H.; Hoyer, B.F.; Jacobi, A.M.; Hansen, A.; Muehlinghaus, G.; Berek, C.; Hiepe, F.; Manz, R.; Radbruch, A.; et al. Generation of migratory antigen-specific plasma blasts and mobilization of resident plasma cells in a secondary immune response. Blood 2005, 105, 1614-1621. [CrossRef]

56. Galson, J.D.; Trück, J.; Fowler, A.; Clutterbuck, E.A.; Münz, M.; Cerundolo, V.; Reinhard, C.; van der Most, R.; Pollard, A.J.; Lunter, G.; et al. Analysis of B Cell Repertoire Dynamics Following Hepatitis B Vaccination in Humans, and Enrichment of Vaccine-specific Antibody Sequences. EBioMedicine 2015, 2, 2070-2079. [CrossRef]

57. Horns, F.; Vollmers, C.; Croote, D.; Mackey, S.F.; Swan, G.E.; Dekker, C.L.; Davis, M.M.; Quake, S.R. Lineage tracing of human B cells reveals the in vivo landscape of human antibody class switching. Elife 2016, 5, e16578. [CrossRef] [PubMed]

58. Neuberger, M.S.; Milstein, C. Somatic hypermutation. Curr. Opin. Immunol. 1995, 7, 248-254. [CrossRef]

59. Storb, U.; Peters, A.; Klotz, E.; Rogerson, B.; Hackett, J. The mechanism of somatic hypermutation studied with transgenic and transfected target genes. Semin. Immunol. 1996, 8, 131-140. [CrossRef]

60. Collins, A.M.; Watson, C.T. Immunoglobulin light chain gene rearrangements, receptor editing and the development of a self-tolerant antibody repertoire. Front. Immunol. 2018, 9, 2249. [CrossRef] [PubMed]

61. Moticka, E.J.; Streilein, J.W. Hypothesis: Nonspecific polyclonal activation of memory B cells by antigen as a mechanism for the preservation of long term immunologic anamnesis. Cell. Immunol. 1978, 41, 406-413. [CrossRef]

62. Blanco, E.; Pérez-Andrés, M.; Arriba-Méndez, S.; Contreras-Sanfeliciano, T.; Criado, I.; Pelak, O.; Serra-Caetano, A.; Romero, A.; Puig, N.; Remesal, A.; et al. Age-associated distribution of normal B-cell and plasma cell subsets in peripheral blood. J. Allergy Clin. Immunol. 2018, 141, 2208-2219.e16. [CrossRef] [PubMed] 